\title{
BMJ Open PREDICT-CP: study protocol of implementation of comprehensive surveillance to predict outcomes for school-aged children with cerebral palsy
}

Roslyn N Boyd, ${ }^{1,2}$ Peter SW Davies, ${ }^{3}$ Jenny Ziviani, ${ }^{2,4}$ Stewart Trost, ${ }^{5}$ Lee Barber, ${ }^{1}$ Robert Ware, ${ }^{6}$ Stephen Rose, ${ }^{7,8}$ Koa Whittingham, ${ }^{1}$ Leanne Sakzewski, ${ }^{1,2}$ Kristie Bell, ${ }^{1,2}$ Christopher Carty, ${ }^{9}$ Steven Obst, ${ }^{1}$ Katherine Benfer, ${ }^{1}$ Sarah Reedman, ${ }^{1}$ Priya Edwards, ${ }^{2}$ Megan Kentish, ${ }^{2}$ Lisa Copeland, ${ }^{2}$ Kelly Weir, ${ }^{6,10}$ Camilla Davenport, ${ }^{1,3}$ Denise Brooks, ${ }^{3}$ Alan Coulthard, ${ }^{8}$ Rebecca Pelekanos, ${ }^{11}$ Andrea Guzzetta, ${ }^{12}$ Simona Fiori, ${ }^{12}$ Meredith Wynter, ${ }^{2}$ Christine Finn, ${ }^{1}$ Andrea Burgess, ${ }^{1}$ Kym Morris, ${ }^{1}$ John Walsh, ${ }^{8,9,13}$ Owen Lloyd, ${ }^{2}$ Jennifer A Whitty, ${ }^{14,15}$ Paul A Scuffham ${ }^{6}$

To cite: Boyd RN, Davies PSW, Ziviani J, et al. PREDICT-CP: study protocol of implementation of comprehensive surveillance to predict outcomes for school-aged children with cerebral palsy. BMJ Open 2017;7:e014950. doi:10.1136/ bmjopen-2016-014950

- Prepublication history and additional material are available. To view, please visit the journal online (http://dx.doi.org/ 10.1136/ bmjopen-2016-014950)

Received 17 February 2017 Revised 11 April 2017 Accepted 27 April 2017

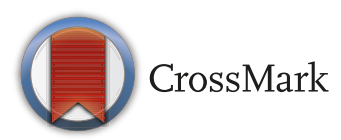

For numbered affiliations see end of article.

Correspondence to Professor Roslyn N Boyd; r. boyd@uq.edu.au

\section{ABSTRACT}

Objectives Cerebral palsy (CP) remains the world's most common childhood physical disability with total annual costs of care and lost well-being of \$A3.87b. The PREDICT-CP (NHMRC 1077257 Partnership Project: Comprehensive surveillance to PREDICT outcomes for school age children with $\mathrm{CP}$ ) study will investigate the influence of brain structure, body composition, dietary intake, oropharyngeal function, habitual physical activity, musculoskeletal development (hip status, bone health) and muscle performance on motor attainment, cognition, executive function, communication, participation, quality of life and related health resource use costs. The PREDICTCP cohort provides further follow-up at 8-12 years of two overlapping preschool-age cohorts examined from 1.5 to 5 years (NHMRC 465128 motor and brain development; NHMRC 569605 growth, nutrition and physical activity). Methods and analyses This population-based cohort study undertakes state-wide surveillance of 245 children with CP born in Queensland (birth years 2006-2009). Children will be classified for Gross Motor Function Classification System; Manual Ability Classification System, Communication Function Classification System and Eating and Drinking Ability Classification System. Outcomes include gross motor function, musculoskeletal development (hip displacement, spasticity, muscle contracture), upper limb function, communication difficulties, oropharyngeal dysphagia, dietary intake and body composition, participation, parent-reported and child-reported quality of life and medical and allied health resource use. These detailed phenotypical data will be compared with brain macrostructure and microstructure using 3 Tesla MRI (3T MRI). Relationships between brain lesion severity and outcomes will be analysed using multilevel mixed-effects models.

Ethics and dissemination The PREDICT-CP protocol is a prospectively registered and ethically accepted study protocol. The study combines data at $1.5-5$ then $8-12$ years of direct clinical assessment to enable prediction
Strengths and limitations of this study:

- The PREDICT-CP prospective cohort study provides comprehensive phenotypical data on a representative cohort of children with cerebral palsy.

- The longitudinal follow-up of this cohort (at 2-5 years and now cross-sectional at 8-12 years) will enable development of prediction models of outcome.

- Brain structure (macrostructure and microstructure at $3.0 \mathrm{~T}$ ) will be compared with comprehensive motor, cognitive and communication outcomes at school age.

- A limitation is that only brain macrostructure at $1.5 \mathrm{~T}$ has been captured from early clinical brain MRI scans as part of clinical practice.

of outcomes and healthcare needs essential for tailoring interventions (eg, rehabilitation, orthopaedic surgery and nutritional supplements) and the projected healthcare utilisation.

Trial registration number ACTRN: 12616001488493

\section{BACKGROUND}

Cerebral palsy (CP) is a disorder of movement and posture secondary to an insult to the developing brain. ${ }^{1}$ The insult is static and permanent and may be the consequence of different factors, including both genetic and environmental causes. Although the insult is static, the consequent symptoms are variable and may change over time. ${ }^{2}$ The disability increases with age and ageing occurs earlier. ${ }^{3}$ Children may have a range of comorbidities, ${ }^{4}$ which are likely to impact outcomes and costs of care. ${ }^{5}$ Based on CP registers, a recent 
systematic review identified that in children diagnosed with CP at 5 years: 3 in 4 were in pain; 1 in 2 had an intellectual disability; 1 in 3 could not walk; 1 in 3 had hip displacement; 1 in 4 could not talk; 1 in 4 had epilepsy; 1 in 4 had a behaviour disorder; 1 in 4 had bladder control problems; 1 in 5 had a sleep disorder; 1 in 5 dribbled; 1 in 10 were blind; 1 in 15 were tube fed and 1 in 25 were deaf. $^{6}$ It is known that peak motor attainment in CP is reached at 8-9 years and tends to plateau before a decline in adolescence. ${ }^{3}$ Secondary musculoskeletal disorders involving muscle, tendons, bones and joints are common as a result of spasticity, muscle weakness and immobility. $\mathrm{CP}$ has substantial lifelong effects on daily function, societal participation and quality of life (QoL) for children and their families. There is a paucity of data on the relationship between physical outcomes and school attainment. ${ }^{7}$ Better prediction of outcomes is important for families and healthcare providers. ${ }^{8}$

In Australia, CP remains the most common physical disability in children with $\approx 700$ infants born each year that will be later diagnosed with $\mathrm{CP}^{9}{ }^{9}$ The overall costs to society of persons with $\mathrm{CP}$ was $\$ \mathrm{~A} 1.47 \mathrm{~b}$ per year $(0.14 \%$ of GDP), with an average annual cost of $\$$ A43431 per individual. ${ }^{10}$ When taking into account the value of lost well-being (disability and premature death), the total costs were $\$ \mathrm{~A} 3.87 \mathrm{~b}$ per year or $\$ \mathrm{~A} 115000$ per person. $\mathrm{CP}$ has a lifetime impact at a total cost of over \$A2M per person. ${ }^{10}$ More recently, in a preschool-aged cohort (CP-Child, National Health and Medical Research Council NHMRC 465128), we have determined a strong relationship between severity of Gross Motor Function Classification System (GMFCS) levels I-V and a stepwise increase in incremental costs of care. ${ }^{5}$

The ability to better predict outcomes has the potential to guide intervention to reduce adverse outcomes (hip dislocation, poor growth, undernutrition or overnutrition, respiratory health complications from oropharyngeal dysphagia (OPD), pain, reduced participation in the community and under attainment at school). Development of prediction models based on early brain structure and function can inform health and social care provision (eg, via the National Disability Insurance Scheme (NDIS)) and provide best practice comprehensive surveillance to allow implementation of timely and effective interventions to achieve optimal outcomes.

Understanding the relationship between specific brain MRI appearance and outcome measures such as motor function is critically important. ${ }^{11}$ Such data may prove invaluable in providing accurate prognostic counselling at the time of diagnosis, as well as potentially guiding the most appropriate treatments tailored to each individual's pattern of CP and type and severity of the brain lesion on imaging. ${ }^{12} \mathrm{~A}$ focus of the majority of epidemiological research is the prevention of $\mathrm{CP}$, which requires clinical outcomes to be correlated with the presumed timing and aetiology of lesions in the developing brain. ${ }^{11}$ Pathological insults in the developing brain cause abnormalities or lesions, which may be detected by brain MRI, and the patterns of these lesions depend on the stage and/ or presumed timing of the injury during brain development. ${ }^{13}$ Using this principle, a qualitative system of classification is established, whereby lesions can be identified as brain maldevelopments (occurring in the first and second trimesters), ${ }^{11}$ periventricular white matter lesions (occurring early in the third trimester and in preterm infants) or grey matter lesions (occurring late in the third trimester and at term). ${ }^{11}$ A systematic review found studies with enough MRI data for subjects to be classified into these presumed lesion timing groups, and in the majority of studies this lesion timing classification was able to be linked to at least one measure of motor outcome. ${ }^{11}$ There are limited data on brain lesion severity, brain microstructure and quantitative comprehensive outcomes. ${ }^{11}$

In the Australian CP-child study, entire birth years of Victorian and Queensland born children with CP across the full spectrum of gross motor abilities were prospectively followed to determine the relationship between the rate and limit of motor development (gross and fine motor function) as related to the nature of the brain lesion. ${ }^{12}{ }^{14}$ Representative population-based data have been reported on i) early development and prediction of hip outcomes ${ }^{15}$ ii) the relationship between brain structure and motor development ${ }^{12}$ and iii) social function ${ }^{16}$ and communication ${ }^{17}$ with cost and health resource use data across the spectrum of functional severity. ${ }^{5}$ The cross-sectional domains of school readiness (mobility, self-care, social function, communication) were reported at school entry. ${ }^{16} 18$

In CP, there is a likely relationship between the severity of the early brain injury on structural MRI (nature, extent, presumed timing), early motor status at 3 years and later outcomes at 8-12 years (motor attainment, musculoskeletal performance, hip displacement). In Sweden, Norway and Scotland, a population-wide surveillance programme (CP-UP) has been implemented for up to 10 years. ${ }^{19}$ Since implementation in Southern Sweden, no child with $\mathrm{CP}$ has had a dislocated hip, ${ }^{19}$ musculoskeletal contractures have been reduced ${ }^{20}$ and nutrition and bone health are monitored. ${ }^{21}{ }^{22}$ National hip surveillance best practice guidelines have been developed and implemented in Australia, ${ }^{23}$ and in Queensland population-wide hip surveillance has been implemented. ${ }^{24}$

The PREDICT-CP study will undertake further comprehensive follow-up of four birth years of children with CP born in Queensland to capture longitudinal data on growth and physical outcomes (motor capacity, muscle and bone health, physical activity, feeding and oropharyngeal function, nutrition), cognition (executive function, educational attainment, communication) and participation, QoL, pain and these are related to costs of healthcare utilisation. The quantitative evaluation of early brain structure on MRI and functional status around 2 years will be compared with these comprehensive outcomes at 8-12 years to build prediction models of CP. Development and implementation of prediction 
models of outcomes are essential for tailoring interventions (rehabilitation, medical management, orthopaedic surgery, nutritional supplements) and in understanding the likely costs of healthcare.

Growth, nutrition and physical activity are important determinants of health outcomes in children with CP. Knowledge oflevelsand patterns of habitual physicalactivity (HPA) for children with CP are important as they have increased risk of inactivity (sedentary behaviour) related illness. ${ }^{9}{ }^{25}$ In addition, poor nutrition and growth may have a secondary impact on body composition, bone health and brain maturation, as well as participation and health-related quality of life (HRQoL) in later childhood. In our overlapping CP-child study of growth, nutrition and physical activity (NHMRC 569605), ${ }^{26}$ we have determined at preschool-age the i) energy requirements, body composition, dietary intake, ${ }^{27-29}$ ii) validation of HPA cut-points, ${ }^{30}$ iii) validation of a modified 3-day weighed food record for the assessment of energy intake ${ }^{29}$ and iv) OPD across the spectrum of functional severity. ${ }^{31} 32$

Our early data on nutritional status ${ }^{29}$ used gold standard measures (doubly labelled water) to determine the energy requirements of preschool-aged children with CP compared with age-matched children with typical development (TD).$^{28}$ Children who were GMFCS III-V had energy requirements $18 \%$ lower than ambulant children and $31 \%$ lower than children with $\mathrm{TD}^{28}{ }^{28}$ with no differences between ambulant children with $\mathrm{CP}$ and children with TD. In addition, energy intake was related to fat-free mass (FFM) index in both children with $\mathrm{CP}$ and children with TD. ${ }^{29}$ Associations were identified between OPD, energy intake and nutritional status after GMFCS level was taken into account. At preschool age, OPD was originally reported in $85 \%$ of our cohort, with a significantly greater proportion of OPD with each increase in GMFCS level. ${ }^{31}$ Following further testing of OPD psychometrics, with the inclusion of a typically developing reference sample, modified cut-points were developed resulting in a revised estimate of $56 \% .{ }^{33}$ Children on full oral intakes that required modification (texture or additional energy and protein) were most at risk of poor growth and nutritional status. ${ }^{31}$

HPA accelerometer cut-points have been determined for sedentary and active behaviour in toddlers with $\mathrm{CP}^{34}$ demonstrating that HPA levels are highly variable within GMFCS levels particularly GMFCS I-II. ${ }^{35}$ The musculoskeletal development of children with $\mathrm{CP}$ has focused on how spasticity interferes with normal muscle growth, and contributes to reduced joint range of motion, increased joint stiffness and muscle weakness. ${ }^{36}$ These factors lead to fixed contractures of the muscle-tendon unit and skeletal deformity that may require orthopaedic surgery. ${ }^{37}$ These secondary alterations progress with age ${ }^{38}$ and contribute to reduced gait speed, increased joint pain and falling, culminating in reduced HPA. ${ }^{39}$ Muscle adaptations begin early $^{37}$ and compared with children with TD vary in the following ways: i) muscle volume is reduced ${ }^{36} 40$; ;i) muscle fascicles are stiffer when passively stretched ${ }^{40}$; iii) muscle fascicles cannot stretch to lengths more favourable for force production ${ }^{42}$ and iv) the Achilles tendon is longer. ${ }^{42} 43$ This effectively means that the ability of muscle to generate force is reduced in children with CP. In ambulant children (GMFCS I-III), the calf muscle (gastrocnemius/soleus) has a major role in forward propulsion during walking/running ${ }^{44}$ and structural/ functional adaptations are a cause of gait limitations. ${ }^{45}$ Characteristics of muscle structural/functional adaptations also vary according to unilateral/bilateral motor distribution. ${ }^{46}$ Lower limb treatments (casting, intramuscular Botulinum toxin A injections) aim to manage these adaptations in the preschool years; however, multilevel orthopaedic surgery is often required at functional attainment (8-11 years) according to a child's gait profile. ${ }^{4748} \mathrm{~A}$ gait profile of ambulant children (GMFCS I-III) combined with muscle properties would provide important information for surgical decision making and prediction of functional outcome. Examination and surveillance of the relationship between muscle structure/function and gait profile to functional capacity/performance, physical activity, bone health, nutritional status and healthcare costs would provide vital information for structuring management plans into later childhood.

The broad aim of the CP-child studies is to implement population-based comprehensive surveillance of children with CP from early diagnosis (at 1.5-3 years) based on brain structure and function (early gross and fine motor, growth, nutrition, HPA, musculoskeletal development) to predict comprehensive outcomes at school age (8-12 years), a time of definitive motor maturation, walking ability, need for orthopaedic intervention and educational attainment. In this extended follow-up of two previous overlapping prospective population-based CP cohorts (followed from 18 to 24 months corrected age to 5 years) across the full spectrum of functional severity (NHMRC $465128^{14}$; NHMRC 569605), ${ }^{26}$ we will re-examine the relationship to severity of brain structure at 8-12 years on diffusion MRI (dMRI in a $3.0 \mathrm{~T}$ MRI scanner). At 8-12 years, healthcare utilisation is likely to be different to preschool-age so that associations between health resource use and a beneficial health/ social outcome can be re-evaluated. The PREDICT-CP child study (NHMRC 1077257) is prospectively registered at ACTRN: 12616001488493.

\section{AIMS AND HYPOTHESES}

The PREDICT-CP study will undertake comprehensive state-wide surveillance (in Queensland) of four birth years of a representative population-based cohort of children with CP. The relationship between brain structure on growth and physical outcomes (motor capacity, muscle and bone health, physical activity, oropharyngeal function, nutrition), cognition (executive function, educational attainment, communication) and participation (HPA, QoL, pain and sleep), will be related to educational attainment and health resource use costs. 


\section{Hypotheses}

1. The location, extent of the brain lesion(s) on semiquantitative MRI (by 2 years) and early motor capacity and performance (1.5-3 years) will predict severity of motor capacity Gross Motor Function Measure (GMFM-66) and performance (6 min walk test (6MWT)), Paediatric Evaluation of Disability Inventory Computer Adaptive Test (PEDI-CAT) at 8-12 years.

2. The rate and limit of gross motor and fine motor development (GMFM-66, Assisting Hand Assessment (AHA), Both Hands Assessment (BoHA)), at 8-12 years will be influenced by the severity of musculoskeletal deformity (ie, slower development will correlate with increased spasticity/contracture, poor muscle function, marked hip displacement, pain, reduced sleep, reduced manual ability).

3. Cognition, executive function, communication and educational attainment will be related to brain lesion severity (location, extent of the brain lesions) on semi-quantitative MRI but not gross and fine motor capacity (GMFCS, Manual Ability Classification System (MACS) ) at 8-12 years.

4. Nutritional status (under/overweight), OPD, body composition (FFM and fat mass (FM) via dual energy X-ray absorptiometry (DXA)), HPA, growth velocity and bone health will be related to the level of GMFCS attainment and will predict: i) higher healthcare utilisation and direct medical costs; ii) lower levels of participation in school, leisure and community and iii) poorer HRQoL.

\section{Study significance}

For children with CP, this unique project will:

1. Quantify the impact of functional severity on medical resource use to inform service provision planning at school age (a period of intensive medical and orthopaedic treatments). From earlier sampling of these cohorts (NHMRC 465128/569605), we have detailed information on the content, dose and compliance, adverse events, medical, surgical and allied health resource use (interventions, medications, equipment) and consequences of outcome (from the age of 1.5 to 5 years). By study completion, we will have lifetime data on all interventions from age 1.5 to 5 years and $8-12$ years, with regular assessments of their functional status/outcomes allowing predictive modelling of outcomes for children with CP.

2. Provide school-age follow-up of this comprehensively studied cohort enabling: i) prediction of outcome (brain structure and multiple outcomes); ii) prognostication on functional, cognitive, communication for school attainment; iii) risk factors for musculoskeletal problems (ie, hip, spine deformity and need for surgery) and iv) health outcomes due to sedentary behaviour, body composition, dietary intake and OPD.
3. Highlight the contribution of poor dietary intake, low levels of HPA and reduced bone health on growth, body composition and fracture risk, taking into account the severity of disability.

4. Define the relationship between HPA levels, motor capacity and muscle performance to predict eventual functional attainment and community performance.

As CP remains the most common childhood physical disability with high lifetime costs, models to predict outcomes and costs of care will inform health provision, social care and tailor data for national funding schemes such as the Australian NDIS.

\section{METHODS}

All children diagnosed with CP, born between 1 January 2006 and 31 December 2009 in Queensland will be invited to participate. These children had participated in two prospective longitudinal cohort studies between the ages of 1.5 and 5 years and will be invited to return at 8-12 years. The inclusion criteria included children with CP defined as a permanent (but not unchanging) disorder of movement and posture that resulted from an insult to the developing central nervous system. The characteristic signs were spasticity, movement disorders, muscle weakness, ataxia and rigidity. ${ }^{14}$ The exclusion criteria included i) children with a progressive or neurodegenerative lesion and ii) children born outside Queensland in the relevant birth years.

\section{Ethics approvals}

There are no known health or safety risks associated with participation in any aspect of the described study. All radiological tests (including anterior-posterior (AP) pelvis, spine as required) and full body and lateral distal femur DXA for body composition and bone health have been reviewed for radiation safety. All families gave written informed consent to participate, and they were able to withdraw their child from the study at any time without explanation, without any penalty from staff at Children's Health Queensland, or any effect on their child's care. Data collected in this study have been stored in a coded re-identifiable form (by ID number).

\section{Ascertainment of the cohort}

Prospective entry of birth years Queensland (born in 2006, 2007, 2008, 2009) who were entered from diagnosis commencing at 18 months and followed until school age (5 years) $(n=245)$ in the Australian CP child study were invited to participate in the PREDICT-CP follow-up study. State-wide recruitment was established in collaboration with the Queensland Cerebral Palsy Register with data collection at tertiary referral hospitals. In cases where the diagnosis of CP was unclear, or where there was a suggestion of a progressive or degenerative course, further investigations (such as metabolic screening) were requested before a diagnosis of $\mathrm{CP}$ was confirmed. Children detected after 18 months of age were entered into 


\begin{tabular}{|c|c|}
\hline $\begin{array}{c}\text { CP-child surveillance } \\
2-5 \text { years }\end{array}$ & $\begin{array}{c}\text { Future Studies } \\
\text { At adolescence and young } \\
\text { adults (13-14 \& 16-18 years) }\end{array}$ \\
\hline $\begin{array}{l}\text { Perinatal \& birth history (CP register) } \\
\text { Classification: CP diagnosis, motor } \\
\text { type, motor distribution } \\
\text { GMFCS, MACS, EDACS, FMS } \\
\text { Brain MRI (semi-quantitative) } \\
\text { Body Structure and Function: } \\
\text { Anthropometry } \\
\text { Musculoskeletal: hip x-ray, spinal } \\
\text { exam, } \\
\text { Feeding (SOMA, DDS, Saliva Scale, } \\
\text { clinical signs of aspiration) } \\
\text { Body composition (BIA) } \\
\text { Dietary intake } \\
\text { Physical activity (Actigraph) } \\
\text { Participation: PEDI-CAT, } \\
\text { HRQOL (CP-QOL) at } 4 \text { years } \\
\text { Health resource use Q (HRU Q) }\end{array}$ & $\begin{array}{l}\text { Classification: GMFCS-ER, MACS, CFCS, FCCS, Viking Speech Scale, } \\
\text { EDACS, motor type and distribution, gait patterns, } \\
\text { Brain MRI at 3T (SMRI, dMRI) } \\
\text { Body Structure and function: } \\
\text { Function (GMFM-66, 6MWT, 10mFWT, MPST) } \\
\text { Bimanual co-ordination (AHA or BoHA) } \\
\text { Gait profile (3DGA) pre/post-orthopedic treatment. } \\
\text { Musculoskeletal: hip x-ray, spinal exam, fracture, pain Q, sleep Q, sexual } \\
\text { maturation (Tanner stage), muscle mechanics 3D ultrasound. } \\
\text { Body composition, bone mineral density (DXA), Sun Exposure Diary, } \\
\text { Vitamin D and Growth Hormone. } \\
\text { Diet (3 day food), BMI, Feed/swallow ability Q, dysphagia (DDS, SOMA } \\
\text { Saliva Scale, clinical signs) } \\
\text { Communication (CELF-4, PPVT, VMPAC) } \\
\text { Physical activity (Actigraph } 5 \text { days) } \\
\text { Participation: PEDI-CAT, PEM-CY } \\
\text { Cognition (RCPM, BRIEF, Connors 3, NAPLAN, AEDI), Behaviour SDQ, } \\
\text { PQ10-child; } \\
\text { HRQOL (CPQOL child, CHU-9D generic), HRU Q. } \\
\text { Parent: CCES, FAD. }\end{array}$ \\
\hline
\end{tabular}

Key: $\mathrm{AHA}=$ Assisting hand Assessment; $\mathrm{AEDI}=$ Australian Early Development Index; $\mathrm{BIA}=$ Bioelectrical Impedance Analysis; BMI=Body Mass Index; BoHA=Both Hands Assessment; BRIEF=Behaviour Rating Inventory of Executive Function; CELF-4=Clinical Evaluation of Language Fundamentals-4; CFCS=Communication Function Classification System; CHU9D=Child Health Utility 9D; CCES=Carer Experience scale; $\mathrm{CP}-\mathrm{QOL}=$ Cerebral Palsy Quality of Life Scale; 3DGA=3 dimensional gait analysis; DDS=Dysphagia Disorders Survey; dMRI=diffusion MRI; DXA=Dual-energy X-ray Absorptiometry; $F A D=$ McMaster Family Assessment Device General Functioning Scale; FMS=Functional Mobility Scale; FCCS=Functional Communication Classification System; EDACS=Eating \& Drinking Ability Classification System; GMFCS-ER=Gross Motor Function Classification System Expanded \& Revised; GMFM-66=Gross Motor Ability Estimator; HRQOL=Health Related Quality of Life; MACS=Manual Ability Classification System; MPST=Muscle Power Sprint test; 6MWT=Six Minute Walk Test; $10 \mathrm{mFWT}=10$ Metre Fast Walk test; NAPLAN=National Assessment Program (Literacy and Numeracy); PEDI-CAT=Paediatric Evaluation of Disability Inventory Computer Adapted Test; PEM-CY= Participation \& Environment Measure for Children and Youth; PQ10-child=Autism Spectrum Quotient child; $\mathrm{RCPM}=$ Raven Coloured Progressive Matrices; SDQ=Strengths \& Difficulties Q; PPVT= Peabody Picture Vocabulary Test; SOMA=Schedule for Oral Motor Assessment; VMPAC=Verbal Motor Performance Assessment for Children.

Figure 1 Summary of surveillance and outcome measures for the PREDICT study.

the study at the time of diagnosis, offered brain MRI at entry and were followed up with serial motor assessments and other outcomes until 5 years.

The recruited sample born in Queensland $(\mathrm{n}=245)$ in the birth years of 2006, 2007, 2008 and 2009 are representative of a population based sample. ${ }^{49}$ The sample is classified according to GMFCS for 2-18 years, a five-level classification system of children's functional gross motor severity. ${ }^{39}$ It is based on self-initiated movements, antigravity postures and motor skills expected in a child aged 5 years. ${ }^{50}$ Children who are independently ambulant are classified as GMFCS I or II, those requiring an assistive mobility device to walk classified as GMFCS III and those in wheeled mobility as GMFCS IV and V. The recruited sample included children who were functioning at 5 years of age at GMFCS level $\mathrm{I}=96(39.2 \%), \mathrm{II}=38(15.5 \%)$, $\mathrm{III}=38(15.5 \%), \mathrm{IV}=35(14.3 \%)$ and $\mathrm{V}=38(15.5 \%)$, of whom 146 were male $(59.6 \%)$, of spastic motor type $208(84.9 \%)$ and unilateral $78(31.8 \%)$ or bilateral 165 $(67.3 \%)$ motor distribution (figure 1). Children will be assessed during their 8-11 birth year at the Centre for Children's Health Research in Brisbane. Comorbidities and need for medical management will be screened.

\section{Procedures}

Children and families participated in previous research projects (NHMRC 569605 and NHMRC 465128) and were born in Queensland will be approached to participate in the current study. After providing informed consent, the child and their caregiver are invited to attend the Children's Health Research Centre and the Lady Cilento Children's Hospital, a tertiary referral centre for 1-2 days visit. All recent medical, surgical and neurological visits that had occurred since their last visit will be screened (from their medical records and by parent report) to confirm any changes in diagnosis of CP, differential diagnosis by neurological assessment (by a paediatrician, child neurologist or paediatric rehabilitation specialist). Experienced allied health researchers performed all motor, upper limb, language and cognitive assessments at the 
visit. Physiotherapists will check range of motion, clinical measures of spasticity, then rate the GMFCS classification, gait pattern and will measure the pelvic and spine radiographs where indicated according to standardised protocols. ${ }^{23}$

\section{Classification measures}

All children with CP at all levels of ability (GMFCS I-V) at $8-12$ years will be classified as mentioned below.

\section{Functional severity}

The GMFCS has internationally established validity, reliability and stability for the classification and prediction of motor function of children with CP aged 2-12 years. ${ }^{50-52}$ It has an acceptable inter-rater and intrarater (testretest) reliability (generalisability coefficients 0.93 and 0.68 , respectively) ${ }^{51}$ Two physiotherapists, trained in the use of the GMFCS, independently observed and classified children in one of five functional categories. ${ }^{50}$

Classifications of gross motor abilities change with age and therefore separate descriptions were used for different age bands. In the present study, the 6-12years descriptions from the extended and revised GMFCS (GMFCS-ER) will be used. ${ }^{53}$ The GMFCS has been correlated with a number of motor scales, as well as CP motor type and distribution. ${ }^{54}$

\section{Motor type and distribution}

Motor type was classified as spastic, dystonic, ataxic, hypotonic, choreoathetosis, mixed CP or unclassifiable according to Surveillance of Cerebral Palsy in Europe guidelines. ${ }^{55}$ Distribution was classified by number of limbs impaired, unilateral and bilateral distribution (hemiplegia, diplegia, triplegia, quadriplegia) by at least two independent raters. The Dyskinesia Impairment Scale ${ }^{56}$ will be undertaken for those participants with a motor-type (primary or secondary) diagnosis of dystonia and/or choreoathetosis. This is an important assessment to measure the motor capacity and function of children with these particular motor types. ${ }^{57}$

\section{Functional performance}

The Functional Mobility Scale ${ }^{58}$ at $5 \mathrm{~m}$ (home), $50 \mathrm{~m}$ (school) and $500 \mathrm{~m}$ (community) ${ }^{58}$ will be used to evaluate functional performance. This is a valid and reliable measure of a child's usual walking ability at three distances ( $5 \mathrm{~m}, 50 \mathrm{~m}$ and $500 \mathrm{~m}$ ), representing their home, school and wider community, respectively. ${ }^{59}$

\section{Gait pattern}

Gait patterns will be classified according to the classification by Rodda and Graham, ${ }^{60}$ which demonstrated validity and reliability. ${ }^{61}$ Gait patterns for bilateral ambulant CP will be classified as either: i) true equinus, ii) jump knee, iii) apparent equinus or iv) crouch gait. For children with unilateral CP, gait patterns were classified according to the classification by Winters $e t a_{l}{ }^{48}$ This classification considered the sagittal plane joint movements: i) type Ifoot drop during swing phase (apparent equinus); ii) type
II-persistent ankle plantarflexion (true equinus); iii) type III-maintained plantar flexion through gait cycle plus limited knee flexion-extension and iv) type IVsimilar to III, plus reduced hip flexion-extension. The classification by Winters et al had good inter-rater reliability using written reports (weighted kappa, $w \kappa=0.76$ ) and videos $(\mathrm{wK}=0.63) .{ }^{61-63}$

\section{Upper limb function}

Upper limb function is classified using the MACS. ${ }^{64}$ The MACS is an international system to classify hand function based on the child's typical performance when handling objects in daily activities. The MACS is a five-level classification of how well children with CP use their hands to handle objects in day-to-day activities. ${ }^{64}$ This classification system was developed for children aged 4-18 years, and has good reliability for use in children as young as 2 years. ${ }^{64}$ The MACS has reported construct validity, and excellent inter-rater reliability (intraclass correlation coefficient $=0.97$ between therapists and 0.96 between therapists and parents) for children with $\mathrm{CP}^{65}$ Children will be classified using the MACS by an occupational therapist in discussion with the child's carer.

\section{Communication function}

Communication function will be classified on three distinct but overlapping systems:

1. Communication Function Classification System classifies children's performance in sending and receiving communicative messages using their typical communication means (considering all communication methods including Augmentative and Alternative Communication). It has been validated in children with CP aged 2-18 years. Reliability between professionals was moderate $(\kappa=0.66)$, professionalparent fair $(\kappa=0.49)$ and test-retest strong $(\kappa=0.82){ }^{66}$

2. Functional Communication Classification System classifies children's performance only in sending communicative messages, and also considers their typical communication (including all communication methods including Augmentative and Alternative Communication). It has excellent inter-rater reliability between professionals $(\kappa=0.94)$ and parentprofessional $(\kappa=0.59){ }^{67}$

3. The Viking Speech Scale (VSS) was used to classify children's speech production. ${ }^{68}$ The VSS is a fourlevel classification system, which can be used to classify speech intelligibility for strangers and unfamiliar conversation partners of children with $\mathrm{CP}$ aged 4 years and above. It has strong content validity, and moderate-to-substantial inter-rater reliability between pairings of speech pathologists, healthcare professionals and parents $(\kappa=0.58-0.81) .{ }^{68}$

\section{Eating and drinking function}

The Eating and Drinking Ability Classification System (EDACS) classifies the eating and drinking abilities of children with CP aged 3 years and above. Classification 
is I-V and describes children's safety and efficiency predominately focusing on food and fluid textures. ${ }^{69}$ The EDACS has strong inter-rater reliability between professionals (ICC $=0.93$ ), but fair reliability between parings of professionals and parents $(\mathrm{ICC}=0.45) .{ }^{69}$

\section{Primary outcomes}

This prospective longitudinal study follow-up has two primary outcomes (hypothesis 1):

1. Gross motor function will be evaluated using the GMFM-66;

2. Brain lesion severity will be assessed using a structured scoring proforma (Fiori scale).

All additional measures are secondary outcome measures (hypotheses 2-4).

\section{Body structure and function measures}

Brain structure on MRI

The American Academy of Neurology practice parameter has recommended that brain MRI should be part of the diagnosis of $\mathrm{CP} .^{70}$ Early MRI at $0-3$ years was classified according to the nature and presumed timing of the lesion ${ }^{11}$ and analysed for brain lesion severity on the semi-quantitative scale of Fiori. ${ }^{8}$ Aetiology of CP was evaluated using MRI (location, nature and structure of the brain lesion). ${ }^{11}$ The brain lesion was classified by three main criteria:

1. The anatomical features of the lesion:

2. localisation by tissue (eg, cortical, white matter, deep grey matter, etc)

3. localisation by region (eg, lobes involved, laterality, etc)

4. extent of lesion (eg, generalised, hemispheric, lobar, etc)

5. The presumed aetiology of the lesion: i) genetic; ii) ischaemic; iii) infective and iv) other.

6. The presumed timing of the insult that caused the lesion:

7. Prenatal by trimester or by stage of brain development;

8. Perinatal;

9. Postnatal.

All MRIs were classified by a neurologist (SF) together with a neuroradiologist (AC) using a standardised method of image evaluation and classification. Following these evaluations, consensus was reached regarding the above three criteria. Based on preliminary data, it was estimated that $>60 \%$ of children currently receiving a diagnosis of CP had early brain MRI as part of their clinical workup. All children $(n=245)$ will be offered a repeat brain MRI at $8-12$ years at $3 \mathrm{~T}$. The majority will have their imaging performed and reported through the Herston Imaging Research Facility, on a Siemens 3.0 T MR scanner. The current minimum imaging protocol for patients with suspected CP consists of axial fast spin echo and coronal fast spin echo sequences and three-dimensional (3D) inversion prepared fast spoiled GRASS sequence. The 3D acquisitions will be reformatted in axial, coronal and sagittal planes, with additional oblique and curved reformatting. Age-specific protocols will be used to maximise the ability to detect cortical and white matter abnormalities at different stages of myelination. All neuroimagings will be reviewed by a neurologist (SF, AG) and a neuroradiologist (AC) familiar with the features of lesions that resulted in CP. This approach is consistent with a clinical practice guideline suggesting that all patients with the label of CP had high-quality MRI on at least one occasion. ${ }^{70}$ MRI scans will be performed predominantly awake, without anaesthesia and after informed consent. Preparation for the MRI will be offered to families in the form of a training DVD explaining the scanner experience and practice in a 'mock scanner' $(0.0 \mathrm{~T})$ where required.

Brain lesion severity will be assessed using a structured scoring proforma ${ }^{8}$ based on the CH2 template, ${ }^{71}$ a highly detailed single-subject T1 template in Montreal Neurological Institute MNI space, which is the international standard for brain mapping (International Consortium of Brain Mapping). Lesions will be transcribed onto the proforma and the following measures obtained: i) number of anatomical lobes involved, ii) number of slices on the template that were affected and iii) size and distribution of the lesion measured by a global lesion score and lesion subscores. The score (maximum of 40) is based on: i) anatomical lobes involved; ii) number of affected slices and iii) size and distribution of the lesion. The number of lobes and slices affected will be the average of summed right and left hemispheres. To calculate total lesion score, each frontal, parietal, temporal and occipital lobes will be first considered in three sections: periventricular, middle and subcortical matter. Each section scored 0.5 if $<50 \%$ of area is involved; or 1 , for $>50 \%$ involvement, with a maximum lobar score of 3. Lobar scores for each hemisphere are summed, with a maximum hemispherical score of 12. The total lesion score is the sum of right and left hemispherical scores (maximum score of 24). A 1-point score (involved/not involved) wis also attributed to 16 anatomical structures including the corpus callosum, the cerebellum and the main subcortical structures. The final maximum score of the scale is, thus, a maximum of $40(24+16) .{ }^{72}$ The Fiori scale method has strong inter-rater and intrarater reliability $^{72}$ and strong construct validity based on dMRI and functional severity in children with unilateral $\mathrm{CP}^{73}$

At 8-12 years, structural MRI (sMRI)-guided and functional MRI-guided dMRI scans suitable for connectivity analyses will be undertaken on the 3T scanner at Herston Imaging Research Facility (or The Lady Cilento Children's Hospital at 3T for children requiring general anaesthesia $\approx 5 \%$ ). dMRI for white matter fibre tracking and whole brain connectomes will be acquired using our published protocol. ${ }^{74}$ The sMRI images will be acquired using an Magnetisation Prepared Rapid Acquistion Gradient Echo(MPRAGE) sequence at an isotropic resolution of $1 \mathrm{~mm}$. dMRI data will be preprocessed to reduce image artefacts, ${ }^{75}$ and the fibre orientation distribution was estimated using constrained spherical deconvolution. ${ }^{76}$ 
Probabilistic tractography will be conducted using MRtrix software and connectivity matrices generated using previously described methods. ${ }^{74}$ Quantitative diffusivity indices Fractional Anisotrophy (FA) and Mean Diffusivity (MD) will be encoded within the connectome to assess reorganisation. ${ }^{74}$ Network-based statistics ${ }^{77}$ will be performed between FA and MD connectomes to identify significant cortical networks associated with neural reorganisation. A second analysis will investigate brain maturation by comparing serial sMRI data acquired around 2 years with scans in the same children at 8-12 years to develop a predictive model of brain structure and functional outcome using spatiotemporal analysis of the longitudinal imaging data. ${ }^{77}$

\section{Clinical history and examination}

Clinical history will be reviewed (see online supplementary Appendix 1: Queensland CP Child Physicians Checklist) to determine:

a. Presence or absence of comorbidities including vision impairment, hearing difficulties, epilepsy;

b. Feeding issues including presence or absence of gastrostomy tube and failure to thrive;

c. Respiratory difficulties including episodes of pneumonia and aspiration.

A comprehensive musculoskeletal examination will be performed by a physiotherapist to record data relating to joint range of movement, leg length difference, bony anomalies, motor type and lower limb muscle spasticity and contracture. ${ }^{78-83}$

\section{Anthropometry}

Anthropometric measures will be collected as described in detail in our published growth, nutrition and physical activity protocol, ${ }^{26}$ including the following:

a. Body mass to the nearest $100 \mathrm{~g}$ using chair scales (Seca).

b. Height to the last completed millimetre with a stadiometer, or, length using a supine measuring board. Where a direct measure of height or length could not be obtained, height will be estimated from knee height or upper arm length using published validated techniques and formulas. ${ }^{84}$

c. Body mass index will be calculated as mass $(\mathrm{kg})$ divided by height $(\mathrm{m})$ squared.

d. Growth and growth velocity (Z-scores of measured or predicted height).

Weight and body mass index Z-scores will be calculated for age and sex according to the Centres for Disease Control and Prevention 2000 growth data. $^{85}$

\section{Gross motor function}

Gross motor function will be evaluated using the GMFM-66 and GMFM- $88^{86}$ by experienced research physiotherapists. The GMFM- 88 assessed a child's motor abilities in lying to rolling, sitting, crawling to kneeling, standing, walking, running and jumping. The GMFM-66 comprised a subset of the 88 items identified (through Rasch analysis) as contributing to the measure of gross motor function in children with CP. The GMFM-66 will provide an overall measure of gross motor function and the GMFM- 88 provides domain scores to explore specific motor skills. ${ }^{86}$

\section{Upper limb performance}

Children with unilateral CP whose manual ability is MACS I-III will be assessed on the school kids AHA, a Rasch measure of effectiveness of impaired hand in bimanual activities. Test-retest reliability was high (ICC 0.98) and there was predictive validity of future assisting hand use. ${ }^{87}$ BoHA will be used for children with bilateral CP who were manual ability MACS I-IV. The BoHA test content was developed by researchers in Norway and Sweden through modification of the AHA test items and by generation of new items. ${ }^{88}$ Associations between BoHA measures and MACS levels showed strong correlation (Spearman's rho: $0.74)$. The person separation ratios (4.36 and 5.19) and the person reliability $(0.95$ and 0.96$)$ for the subscales indicated that the children's hand function could be separated into six and seven ability levels. ${ }^{88} \mathrm{BoHA}$ is the first observation-based assessment of effective use of the hands in bimanual activities for children with bilateral CP.

Hand dominance will be assessed using the Edinburgh Handedness Inventory laterality quotient. ${ }^{89}$ The Edinburgh Handedness Inventory questionnaire consists of 10 items regarding hand preference (right or left) in performing a number of everyday tasks requiring one (eg, writing, drawing, throwing and using scissors) or two hands (eg, using a broom or opening a box). The laterality quotient is calculated using the following formula: laterality quotient $=$ (right hand-left hand $/$ (right hand+left hand) $\times 100$ ). The Edinburgh Handedness Inventory is included to objectively determine upper limb dominance. This classification system consists of a table that requires the participants to indicate which hand they use to perform a selection of everyday tasks.

Stereognosis relates to a participant's ability to perceive and recognise objects by using only tactile information ${ }^{9091}$ and will be assessed on the impaired and unimpaired limbs, using the approach described by Sakzewski et al. ${ }^{91}$ Participants will be required to identify objects placed in their hand, without any visual cues. A total of nine objects will be placed in the hand one at a time. Three familiar objects (teaspoon, key, peg) and six similar matched objects (safety pin and paperclip; pen and pencil; coin and button) will be used. With vision occluded, participants are presented with each item. If a participant is unable to grasp, manipulate or release an object, the occupational therapist assisted the participant and will move the object for them within their hand. A corresponding set of items will be used to allow participants to identify the object in order to minimise any errors due to incorrect naming of the object. Scores ranged on a scale from 0 to 9, where participants scoring below 9 are considered to have impaired stereognosis. ${ }^{90} 91$ 
Radiological measures of hip displacement and spine

Hip surveillance, including AP pelvis X-ray, is recommended for all Australian children with CP to facilitate early detection and treatment of severe or progressive hip displacement. ${ }^{19} 9293$ The migration percentage is widely accepted as the gold standard measure in hip surveillance, ${ }^{78} 94$ measuring femoral head displacement. ${ }^{95}$ Other measures included the Acetabular Index, assessing acetabular dysplasia, ${ }^{96}$ the Hilgenreiner's epiphyseal angle (HEA) ${ }^{96}$ and the femoral neck-shaft angle. ${ }^{9597}$ The $\mathrm{HEA}^{96}$ is a radiographic measure describing the proximal femoral epiphysis and has been previously applied to assessment of coxa valga, ${ }^{98} 99$ but may offer prognostic information for hips at risk in CP. The HEA represents the acute angle between a line drawn parallel to and through the proximal femoral epiphysis and Hilgenreiner's line. ${ }^{81} 82$ Physiotherapists will perform a clinical examination of spinal alignment and mobility to screen for evidence of a potential scoliosis or kyphosis. Where indicated, an AP spine radiograph (for scoliosis) or lateral (for kyphosis) will be performed. Spines where scoliosis was evident will be measured according to the Cobb angle. ${ }^{100}$

\section{Body composition and bone health}

Body composition measures and bone parameters will be acquired using a Lunar Prodigy DXA (GE Medical Systems, LUNAR, Madison, Wisconsin, USA). Body composition measures included FM (g) and FFM (g). Bone parameters include areal bone mineral density (g/ $\mathrm{cm}^{2}$ ) and bone mineral content $(\mathrm{g})$ for all total body, bilateral proximal and lateral distal femur sites. The lateral distal femur is a common site of fracture, ${ }^{101} 102$ with the technique previously described, ${ }^{102}$ and measurements are reproducible in children with CP. ${ }^{103104}$ The analysis involves creating three regions of interest, each containing different proportions of trabecular and cortical bone with results for each region of interest (ROI), therefore, treated independently. ${ }^{101}$ Additionally, the proximal and distal femoral sites are used to calculate bone mineral apparent density $\left(\mathrm{g} / \mathrm{cm}^{3}\right)$, derived from the projected bone area $\left(\mathrm{cm}^{2}\right)$ to provide an approximation of volumetric BMD. ${ }^{105}$ All scans in this research are a 'one-off' occurrence, with the total radiation dose for these five DXA scans being $<15 \mu \mathrm{Sv}^{106}$ This is equivalent to approximately 1-2 days natural background radiation exposure, and only equivalent to $3 \%$ of the dose constraint limit for children as research volunteers, up to the age of 18 years. ${ }^{106}$ The total estimated time for all DXA scans is $30 \mathrm{~min}$, performed at the University of Queensland Children's Nutrition Research Centre.

\section{Fracture rate}

Fractures will be diagnosed radiologically. Parents will report by telephone within 24 hours of fracture occurrence and will bring X-ray films and details of management to their study visit. Vertebral fracture will be diagnosed on lateral X-rays of the thoracic and lumbar spine when indicated. Children who were GMFCS III-V will undergo thoracic and lumbar spine (AP/lateral) at 8-12 years if there were clinical signs of fracture and/or scoliosis/kyphosis. Radiographs are minimised to reduce the radiation exposure.

\section{Sexual maturation}

Legal guardians of participants will be provided with standardised Tanner stage puberty diagrams, and parents will be asked to evaluate the child's current pubertal stage. ${ }^{107}$ Parental pubertal assessment will be reviewed by a physician for precocious puberty. In cases of precocious puberty, a left hand/ wrist X-ray will be conducted to determine the bone age and relative skeletal maturity of children. The bone age will be used to determine if the CP condition is interfering with the proper growth and bone development of the child.

\section{Pain}

Children will complete the Paediatric Pain Questionnaire (PPQ) with adult help if required. ${ }^{108}$ The PPQ asks children to report their pain now (severity, type and location), as well as the severity of the worst pain they had in the previous week. The PPQ's visual analogue scale for pain rating provides a valid and stable measure of pain intensity in children and adolescents with chronic musculoskeletal pain. ${ }^{109}$

\section{Three-dimensional gait analysis and in vivo muscle mechanics}

A full 3D gait analysis, including synchronised measurement of muscle activation using electromyography (EMG) and calf muscle mechanics using two-dimensional (2D) ultrasound, will be performed for all children functioning at GMFCS I-III. Participants will walk unaided and barefoot at a self-selected speed over a level walkway $(10 \mathrm{~m}$ in length) with four force platforms embedded in the laboratory floor in the centre of the walkway. Reflective markers will be attached to the trunk, pelvis and lower limbs according to the modified 'Plug in Gait' marker set, with additional clusters of three markers on each thigh and shank segment, and a marker on the fifth metatarsal head. ${ }^{110}$ Marker trajectories will be recorded at $100 \mathrm{~Hz}$ using an 10-camera, 3D motion capture system (Vicon Motion Systems, Oxford, UK) and ground reaction force data will be acquired at $1 \mathrm{kHz}$ using four $510 \mathrm{~mm} \times 465 \mathrm{~mm}$ force platforms (AMTI, Watertown, Massachusetts, USA) arranged in series. Lower limb muscle activations of the rectus femoris (RF), medial hamstrings $(\mathrm{MH})$, medial gastrocnemius (MG), lateral gastrocnemius (LG), soleus (SOL) and tibialis anterior (TA) is recorded for both legs at $1 \mathrm{kHz}$ using a wireless surface EMG system (Aurion ZeroWire, Milan, Italy). Raw EMG signals will be highpass filtered (Butterworth, zero-lag, fourth order, $30 \mathrm{~Hz}$ ) to remove movement artefact, full wave rectified and low passed filtered (Butterworth, zero-lag, fourth order, $6 \mathrm{~Hz}$ ), and interpolated to 101 points per cycle. Non-negative matrix factorisation will be applied to extract muscle synergies, ${ }^{111}$ which represented neuromuscular 
control during gait. Whole body 3D gait kinematics, joint moments at ankle, knee and hip joints and musculotendinous lengths for MG, LG, SOL, RF and MH will be computed across at least five trials using OpenSim ${ }^{112}$ and normalised to length in standing. The Gait Profile Score ${ }^{113}$ will be calculated as an index of overall gait pathology. A digital output signal from the ultrasound system will be used to synchronise acquisition of all 3D marker, force plate and EMG data.

Two-dimensional B-mode ultrasound will be used to examine MG and SOL muscle function during walking by attaching a flat ultrasound transducer (LV7.5/65/64D, Telemed Echo Blaster 64 EXT-1T, Vilnius, Lithuania) to the surface of the skin above the MG muscle and recording muscle fascicle length and pennation angle changes, as described previously. ${ }^{114}$ Muscle fascicle behaviour during walking will be analysed using a semi-automatic process, which has been shown to be highly repeatable (coefficient of multiple correlation 0.88). ${ }^{115}$ The average of five complete strides will be used in the analysis for each participant to ensure the overall reliability of muscle fascicle length data. ${ }^{116}$ Freehand 3D ultrasound will be used to measure muscle size and structure of the lower leg muscles: MG, LG, SOL and TA. ${ }^{117}$ This method of 3D ultrasound is valid (within $1.3 \%$ ) and reliable (ICC $>0.99$ ) for measuring gastrocnemius muscle volume and length in vivo. ${ }^{117}$ Calf muscle physiological cross-sectional area will be measured as the ratio of muscle volume muscle fascicle length, corrected for fascicle pennation angle.

\section{Activity limitations}

The following measures of activity limitations for functional capacity will be performed for ambulant children (GMFCS I-III) at $8-11$ years $(\approx \mathrm{n}=172)$.

\section{6 min walk test}

This simple, submaximal test measures the distance walked over $6 \mathrm{~min}$, providing information about endurance during functional activities. ${ }^{118}$ The 6MWT has excellent test-retest reliability $(\mathrm{ICC}=0.98)$ in $\mathrm{CP} .{ }^{119}$ Percentile curves have been created on 1445 children with TD aged $7-16$ years. ${ }^{120}$ The test will be performed according to guidelines of the American Thoracic Society on a $10 \mathrm{~m}$ course. ${ }^{121}$

\section{Muscle power sprint test}

The muscle power sprint test (MPST) provides an estimate of anaerobic power. ${ }^{122}$ The MPST requires participants to complete six $15 \mathrm{~m}$ runs as fast as possible with $10 \mathrm{~s}$ rest between each lap. Power output is calculated as the product of body mass and distance, divided by time. ${ }^{122}$ The MPST has been validated against the Wingate Anaerobic cycling test, ${ }^{123}$ and has excellent test-retest reliability (ICC 0.98) in children with CP. ${ }^{122}$

\section{$10 \mathrm{~m}$ fast walk test}

The $10 \mathrm{~m}$ fast walk test $(10 \mathrm{mFWT})$ is a test of maximal walking speed over a distance considered the minimum for functional ambulation. The $10 \mathrm{mFWT}$ has moderate test-retest reliability in children with CP (ICC 0.81). ${ }^{124}$

\section{Lower limb functional strength}

Thirty second repetition maximum $\left(\operatorname{rep}_{\max }\right)$ of functional strength exercises (including sit-to-stand, lateral step-ups and half-kneel to stand) will be tested according to published recommendations. ${ }^{125}$ Functional strength tests demonstrate acceptable inter-tester reliability (ICC $\geq 0.91$; coefficient of variation (CV) $12.1 \%-22.7 \%$ ) in children with CP. ${ }^{125}$ For each lower limb functional strength exercise, participants will be given verbal and visual instructions as well as two practice repetitions prior to testing. The exercises were assessed in the following order: sit-to-stand, lateral step-up dominant leg, lateral step-up non-dominant leg, half-kneel to stand dominant and half-kneel to stand non-dominant. Participants will be given verbal encouragement throughout. Participants will be given $180 \mathrm{~s}$ rests between exercises. If a participant does not complete an exercise while performing the practice attempts, they will be assigned a score of 0 and will not proceed to testing.

\section{Habitual physical activity}

Triaxial accelerometers (ActiGraph GT3X+, Pensacola, Florida, USA) will be used to evaluate the frequency, intensity and duration of physical activity. ${ }^{126}$ ActiGraph accelerometers have evidence of validity and interinstrument reliability in children with TD compared with heart rate monitoring, direct observation, indirect calorimetry, whole-room calorimetry and doubly labelled water. ${ }^{26}$ The ActiGraph has been validated for measurement of physical activity intensity in adolescents with CP using oxygen uptake as the criterion measure. ${ }^{127} 128$ ActiGraphs will be fitted during assessment and worn during waking hours for 7 days. ${ }^{126}$ Participants' caregivers completed a 7-day physical activity monitor log to record wear and non-wear times (see online supplementary Appendix 2). Stored data will be uploaded to an excel macro to determine daily wear time, average counts per min, daily time spent in sedentary, light, moderate and vigorous activity. Counts will be classified using established cut-points for children with CP. ${ }^{128}$

\section{Blood samples for growth hormone and vitamin D}

Blood will be collected and tested for hormones and other markers required for optimal growth, bone and metabolic health. Specifically, these tests are liver function, kidney function, full blood count, insulin-like growth factor-1, thyroid hormone, parathyroid hormone, vitamin D3, calcium, phosphate and iron studies. As described above, these parameters of growth, bone health and body composition are often altered in children with $\mathrm{CP}$ and are related to gross motor function classification, body composition, growth velocity and nutritional status. Blood tests are optional and consent will be obtained from the parent and assent from the child where possible. Blood samples will be collected by qualified phlebotomists, who 
are familiar with collecting blood from paediatric subjects using their standard procedures. Where preferred, samples will be collected under general anaesthesia, if a patient undergoes an unrelated and non-emergency surgical procedure (eg, orthopaedic surgery, Botulinum toxin A injections, MRI under anaesthesia). Parents of participants will be advised if these results fall outside the relevant reference ranges in relation to age, gender and pubertal status. Parents will provide informed consent for information to be provided to their treating clinician who will take responsibility for ongoing care and follow-up.

\section{Dietary intake}

Dietary energy intake will be recorded using a 3-day weighed food record as validated ${ }^{29}$ using our published methods. ${ }^{26}$ Food records will be analysed using FoodWorks. Mean energy intake will be expressed as megajoules per day and as a percentage of age-specific and gender-specific recommendations. ${ }^{129}$

\section{Vitamin D intake}

A food frequency questionnaire will be completed by parents/caregivers to determine the habitual intake of vitamin D-containing foods by the participants. The questionnaire consists of a table that requires parents to tick a frequency box and record the brand of a simple list of foods. ${ }^{130}$

\section{Sun exposure}

A 7 day sun exposure diary will measure daily sun exposure in the participants to measure ultraviolet radiation (UV) exposure for vitamin D adequacy. Each day, participants will record the amount of time spent in the sun during each 1 hour interval $(0,<15,15$ to $<30,30$ to $<45$ or 45-60 min) between 05:00 and 19:00 hours. Clothing cover (based on a clothing cover guide provided with the dairy) and use of sunscreen (frequency and application site) using established methodology will also recorded ${ }^{131}$ (see online supplementary Appendix 3). It is proposed that the sun exposure diary is done at the same time as the physical activity monitor record to lessen the burden on the participants. Sun exposure diaries will be done within 2 weeks of serum vitamin D levels being collected, to allow for meaningful interpretation of sun exposure and vitamin D levels.

\section{Oropharyngeal dysphagia}

OPD (feeding and swallowing difficulties) will be evaluated during a digital video-recorded snack of $20 \mathrm{~min}$. Children will be presented with three standardised boluses of five textures; puree, semi-solid, chewable, tough chewable and fluid. The following measures will be used to rate the mealtime:

1. The Dysphagia Disorders Survey (DDS)—part 2 consists of a series of binary judgements on eight ingestion functions across the oral preparatory, oral, pharyngeal and gastro-oesophageal phases (maximum raw score of 22). The DDS has good reliability ${ }^{132} 133$ and convergent validity. ${ }^{132-136}$
2. The Schedule for Oral Motor Assessment (SOMA) consists of seven oral motor challenge categories corresponding to four food textures and three fluid utensils. The SOMA has been validated on 127 young infants; 58 comparison children with typical oral skills, 56 with non-organic failure to thrive (aged 8-24 months) and 13 children with CP and overt feeding difficulties (aged up to 42 months). ${ }^{137}$ It has strong inter-rater reliability ( $\kappa=1.0$ in $68 \%$ of fluid category items and $58 \%$ of food category items) and test-retest reliability between boluses ( $\kappa=1.0$ in $84 \%$ of items). ${ }^{137}$

3. Observations of 16 clinical signs suggestive of pharyngeal phase impairment (eg, cough, gurgly phonation, wet respiration) will be rated premealtime and postmealtime by a trained researcher, and rated according to each food/fluid texture from video by a speech pathologist. ${ }^{138-140}$

4. The Thomas-Stonell and Greenberg scale will be used premealtime and postmealtime to rate saliva loss. ${ }^{141}$ This consisted of two observational ordinal scales (15 ), based on severity and frequency of loss.

5. The Cerebral Palsy Child Feeding Questionnaire, used in the CP-Child Study ${ }^{142}$ gathered information on the child's typical mealtime performance based on parent report, which supplemented the data obtained from the participant's clinical feeding assessment.

6. The Feeding/Swallowing Impact Scale (FS-IS) addresses questions of carer QoL and how to incorporated into the economic evaluation. The FS-IS is a validated tool to measure the impact of caring for a child with dysphagia and concerns on caregiver QoL. ${ }^{143}$ It is an 18-item, parent questionnaire divided into three subsections: i) daily activities; ii) worry and iii) feeding difficulties. The tool was validated on the caregivers of 164 children (median age 14 months, mean: $32 \pm 44$ months) with varying comorbidities including prematurity (born $<37$ weeks) in $66(40 \%)$ children, $144(88 \%)$ were medically complex with conditions in more than one diagnostic-based category and 77 (47\%) of children had feeding tubes. ${ }^{143}$

\section{Communication}

All children will have language assessed using the core language subtests of the Clinical Evaluation of Language Fundamentals screener (CELF-4), ${ }^{144}$ in addition to the Peabody Picture Vocabulary Test (PPVT). The CELF-4 is a criterion referenced assessment of language skills in children aged 5-21 years, with Australian norms available. Children who were non-verbal only will complete the receptive subtests of the CELF-4 and the PPVT. Children unable to participate in standardised assessment (eg, due to significant cognitive, visual or motor limitations) will have language classified using the Triple $\mathrm{C}$, a parent-reported observational assessment. Communication performance will be indicated by parents on a communication questionnaire developed for this study (see online 
supplementary Appendix 4), which includes information on augmentative and alternative communication system type, use, frequency and access. ${ }^{144}$

Speech production will be assessed using the verbal motor production assessment for children (VMPAC). The VMPAC $^{145}$ is a diagnostic tool for the systematic assessment of neuromotor integrity of the motor speech system validated in 1434 children aged 3-12 years ${ }^{146}$. The following subtests of the VMPAC will be administered:

1. Oromotor production in word sequences and sentences (six items): items consisted of three-word and four-word sequences and five-word sentences. These items are designed to evaluate the child's ability to sequence oromotor movements across different plane within a linguistic context.

2. Oromotor production in connected speech and language (five items): this subtest assesses the child's motor control (eg, motor precision) as it varied in the context of higher level language formulation.

3. Oromotor production in automatic verbal sequences (one item): this subtest allows evaluation of speech characteristics including pitch, resonance, vocal quality, loudness, prosody, intonation and rate during production of an automatic speech task (eg, counting to 10 , saying the alphabet).

4. Oromotor production in word sequences and sentences (six items): items consisted of three-word and four-word sequences and five-word sentences. These items are designed to evaluate the child's ability to sequence oromotor movements across different plane within a linguistic context. Oromotor production in connected speech and language (five items): this subtest assesses the child's motor control (eg, motor precision) as it varied in the context of higher level language formulation.

5. Oromotor production in automatic verbal sequences (one item): this subtest allows evaluation of speech characteristics including pitch, resonance, vocal quality, loudness, prosody, intonation and rate during production of an automatic speech task (eg, counting to 10 , saying the alphabet).

Paediatric evaluation of disability inventory computer adapted test Performance of self-care will be evaluated using the parent-reported PEDI-CAT for the domains of self-care, mobility and social functioning using scaled scores (Rasch), which have good validity and reliability. ${ }^{147}$ The PEDI-CAT was developed on the basis of the original PEDI. ${ }^{148}$ Scaled scores (possible range 20-80) for each domain provide an indication of the child's performance along a continuum of item difficulty and are most suitable for research. ${ }^{147}$ Scaled scores give more precise results in the extreme ranges than normative standard scores. ${ }^{147}$ Scaled scores are recommended to track functional progress in children who are substantially delayed. $^{147}$

\section{Participation and environmental measures}

Participation and environment measure for children and youth

The participation and environment measure for children and youth (PEM-CY) is a parent-reported instrument that examines participation and environment across three settings: i) home; ii) school and iii) community. ${ }^{149}$ There are 10 items in the home section, 5 in the school section and 10 in the community setting. For each item, the parent is asked to identify how frequently (over the past 4 months) the child has participated (eight options: daily to never); how involved the child typically is while participating (five-point scale: very involved to minimally involved) and whether the parent would like to see the child's participation in this type of activity change (no or yes, with five options for the type of change desired). For each setting, the parent is then asked to report on whether certain features of the environment make it easier or harder for the child to participate. The PEM-CY has reported moderate-to-good internal consistency $(0.59$ and above) and test-retest reliability (0.58 and above) in a population of children (aged 5-17 years) with and without disabilities residing in the USA and Canada $(n=576) .{ }^{149}$ The PEM-CY will be collected using either a paper or online questionnaire format to gain an understanding of the participation of children and adolescents and the impact of environmental barriers and facilitators.

\section{Strengths and Difficulties Questionnaire}

The Strengths and Difficulties Questionnaire ${ }^{150} 151$ is a 33-item parent-rated questionnaire that is used to assess parents' perceptions of prosocial and difficult behaviours in their child or child adjustment. Parents responded to 25 questions about their child's behaviour in the last 6 months using a three-point Likert scale (ie, ' 0 ' $=$ not true to ' 2 '=certainly true). These 25 questions are combined to create five subscales of: frequency of emotional symptoms; conduct problems; inattention/hyperactivity; peer problems and prosocial behaviour (eg, 'considerate of other people's feelings'). A total score for each scale $(0-10)$ and overall total difficulties score $(0-40)$ is calculated, with higher scores indicating more distress on all scales except prosocial behaviour. Scores of 17 or above for the total difficulties scale are used as a clinical cut-off point. Scores from the five subscales and the overall difficulties scale will be used as a measure of the child's psychological functioning. The overall total difficulties score has been demonstrated to have moderate-to-high internal consistency (Cronbach's $\alpha=0.73-0.82$ ) and testretest reliability $(\mathrm{r}=0.77-0.85) .{ }^{152}$

\section{School attainment}

The Australian Early Development Index is a population measure of development at school entry, assessing school readiness. ${ }^{153}$ The National Assessment Program-Literacy and Numeracy (NAPLAN) measures literacy/numeracy achievement and individual reports will be obtained from families as a measure of early school achievement. ${ }^{154}$ Where applicable, consent will be obtained from parents 
to the release of NAPLAN result by the Queensland Curriculum and Assessment Authority.

\section{Cognition}

Raven's coloured progressive matrices

The Raven's coloured progressive matrices (RCPM) is an assessment of non-verbal intelligence for children aged 5-11 years with intellectual delay or physical disability. It consists of 36 items $(15-30 \mathrm{~min})$ of increasing difficulty in which the child has to complete a pattern. RCPM has validity with $\mathrm{CP}$ and Australian norms. ${ }^{155156}$ In 618 Australian children (aged 6-11 years), the RCPM had good internal consistency $(0.76-0.88)$ and split-half reliability $(0.81-0.90) .{ }^{156}$

\section{Behaviour rating inventory of executive function}

The behaviour rating inventory of executive function is a parent completed 86-item measure of executive function in their child's everyday life, yielding two scores: i) the behavioural regulation index and ii) the metacognition index to form a global executive composite score. Both scores can be converted into $\mathrm{T}$ scores with $\geq 65$ indicative of dysfunction. ${ }^{157}$ It has good convergent and divergent validity with Child Behaviour Checklist and the Behaviour Assessment System for Children, ${ }^{158}$ high internal consistency, (Cronbach's $\alpha=0.80-0.98$ ) for parent form. ${ }^{158159}$

\section{Attention and executive functioning}

\section{Conners three parent short form (Conners 3 )}

The Conners three parent short form (Conners 3) ${ }^{160}$ is a thorough assessment of attention deficit hyperactivity disorder (ADHD) and its most common comorbid problems and disorders in children and adolescents aged 6-18 years. The Conners 3 will be completed by the participant's parents or guardian and consisted of 110 statements and takes approximately 20 mins to complete. Parents or guardians rate each statement using a four-point scale ranging from ' 0 , not true at all (never, seldom)' to '3, very much true (very often, very frequently)'. The Conners 3 measures the seven key areas of inattention, learning problems, aggression, family relations, hyperactivity/ impulsivity, executive functioning and peer relations. Raw scores are converted into $\mathrm{T}$ scores based on a large representative normative sample based on the US consensus data. In addition, the Conners 3 calculates $\mathrm{T}$ scores for symptom scales including ADHD hyperactive/Impulsive, ADHD combined, oppositional defiant disorder, ADHD inattentive and conduct disorder. Both internal consistency coefficients $(\alpha=0.83-0.94)$ and test-retest reliability $(\mathrm{r}=0.52-0.94)$ were good for the Conners 3 parent version total sample age range. ${ }^{160}$

\section{The autism spectrum quotient-child}

The autism spectrum quotient-child (AQ10-child) is a 10-item parent-reported autism screening measure for children aged 4-11 years. ${ }^{161}$ It was developed from the Quantitative Checklist for Autism in Toddlers. Parents respond to the 10 items on a four-point Likert scale from definitely agree to definitely disagree. Items will be summed and the scale had a cut-off of 6 . It had high internal consistency (Cronbach's $\alpha=0.90$ ), sensitivity $(0.95)$ and specificity $(0.89){ }^{161}$

\section{Sleep Disturbance Scale for Children}

Sleep disorders are up to four times more common in children with CP compared with the general population and are linked to both physical (total body involvement, severe visual impairment) and environmental factors (singleparent household, bed-sharing). The most commonly reported disorders include difficulty initiating and maintaining sleep, sleep-wake transitions and sleep breathing disorders. ${ }^{162}$ The Sleep Disturbance Scale for Children is a 27-item parent-reported questionnaire that assesses sleep disturbance in children within the past 6 months. ${ }^{163}$ Each item is responded to on a five-item Likert scale with higher values representing greater clinical severity. Items are summed to produce a total score and six subscores representing different facets of sleep disturbance: disorders of initiating and maintaining sleep, sleep breathing disorders, disorders of arousal, sleep-wake transition disorders, disorders of excessive somnolence and sleep hyperhidrosis. It has good internal consistency (Cronbach's $\alpha=0.71-0.79)$, test-retest reliability $(r=0.71)$ and discriminative validity in distinguishing between clinical and community samples. ${ }^{163}$

\section{QoL measures}

\section{Condition-specific QoL measures}

The cerebral palsy quality of life-child assesses well-being across multiple domains using parent-report (aged 4-12 years) and child self-report from 9 years. ${ }^{164-166}$ Psychometrics are excellent with Cronbach's $\alpha$ 0.74-0.92 parent-proxy report and $0.80-0.90$ child self-report. Test-retest was adequate (ICC $0.76-0.89$ ) and moderately correlated with health $(\mathrm{r}=0.30-0.51) .{ }^{164-166}$

\section{Generic QOL}

The Child Health Utility-9D is a generic instrument for children aged 7-11 years for which there is an algorithm to give a single preference-based utility index for health states (giving a single generic preference-based indicator of each individual's health state), making the data amenable for economic evaluations for interventions. ${ }^{167-170}$ The EuroQoL five-dimensional descriptive system (EQ-5D-5L) is a generic instrument designed to describe and value health based on five dimensions: mobility, self-care, usual activities, pain/discomfort and anxiety/depression. ${ }^{171}$ The EQ-5D-5L will be completed by the parent/caregiver, about themselves and scored using the Australian algorithm. ${ }^{172} 173$ This is relevant (alongside the CES) to address questions such as carers QoL and how to incorporate it into economic evaluation.

\section{Demographic questionnaire}

Parents will be required to complete questionnaires related to various aspects of their child's development/ progress including participation, feeding, HRQoL, sleep, 
fracture history, pain, executive functioning in everyday life, clinical comorbidities including epilepsy, psychological functioning, treatments and interventions the child has received since the completion of the CP-child projects (see online supplementary Appendix 5). The socioeconomic status of Australian families will be classified into tertiles using scores on the Socioeconomic Indexes for Areas Index of Relative Disadvantage. ${ }^{174}$

\section{Parent questionnaires}

Carer Experience Scale

The Carer Experience Scale (CES) will be completed by primary caregivers at their study visit. This validated measure of care-related QoL has six domains (activities, support, assistance, fulfilment, control and relationship with the care recipient) and takes approximately $3 \mathrm{~min}$ to complete. ${ }^{175}$ The CES is scored from an algorithm derived from preferences of the general population and can be used to value carer outcomes in economic evaluation using index values. ${ }^{176}$

The McMaster family assessment device general functioning scale Caring for a child with a disability can have an impact on the health and functioning of the caregiver and family unit. ${ }^{177}$ The family assessment device is a 12-item measure of general family functioning. ${ }^{178}$ Each item is rated on a four-point Likert scale from strongly agree to strongly disagree and the items are summed to create the total score. It has good internal consistency (Cronbach's $\alpha=0.92$ ), construct validity and reliability. ${ }^{178-180}$

\section{Monitoring of resource use and direct costs of treatments}

To determine the relationship between motor prognosis and healthcare costs, resource use and direct costs of treatment will be monitored using the Health Resource Use questionnaire ${ }^{14}$ (see online supplementary Appendix 6). Associations between costs (dependent variable) and all other outcome variables (independent variables) including those related to growth, body composition and HPA will be assessed, with adjustment for confounders such as brain lesion severity. Health-related resource use data will be collected including therapy frequency and duration (traditional/alternate), hospital admissions, GP and medical specialist visits, medications (eg, Botulinum toxin A) and equipment (eg, orthoses). Data will be collected via questionnaire, ${ }^{14}$ supplemented by consented access to individual hospital, Medical Benefits Scheme (MBS) and Pharmaceutical Benefits Scheme (PBS) records. Standard cost sources (MBS, PBS) will be used to apply unit costs to resources. Statistical approaches, which consider the likely skewed distribution of cost data (such as generalised linear models, with extensions to allow for correlations in the data across observations from multiple time points from each individual as described below), and diagnostic-related group costs for admissions to hospital) will be employed. As this cohort is embedded in a state-wide clinical service, we have consistency of interventions based on best practice guidelines for Botulinum toxin A, bone health and hip surveillance generalisable across Australia. All children are offered best practice treatment across the state-wide service.

\section{Data analysis plan}

A comprehensive database has been established for all data collection, including clinical measures, MRI scoring and questionnaires so that it is entered prospectively at the time of each assessment. Summary reports are automatically generated from the database to report back to families and treating clinicians after each visit. Biostatistics methods proposed in this study include analysis of binary outcomes in longitudinal studies using weighted estimating equations (eg, presence of comorbidities); multilevel mixed-effects models of longitudinal binary outcomes (eg, GMFCS levels) and generalised estimating equations for ordinal data.

\section{Sample size}

We assume $95 \%$ of the total sample of 245 children consent to the PREDICT-CP programme (we have $98 \%$ retention in NHMRC 465128, 569605). For Hypothesis $1,{ }^{1}$ assuming between-GMFCS group and within-child variability in GMFM-66 were similar to findings by Rosenbaum et $a l,{ }^{181}$ we were able to detect significant between-group differences in GMFM-66 scores at 8-12 years according to the primary predictor variable of initial GMFCS group (aged 2-5 years) with $>80 \%$ power and alpha $=0.05$ for each pairwise comparison. For comparisons between MRI classification at 2 years and GMFM-66 at 8-12 years, then based on GMFCS and MRI data from NHMRC 465128 (where GMFCS I=30\%, II$\mathrm{V}=16 \%$ each and white matter injury $=43 \%$ ), assuming that GMFM- 66 at $8-12$ years has a SD of 7 units within each GMFCS group, ${ }^{181}$ we are able to detect a difference between children with white-matter damage and other MRI pattern types of $\geq 4.6$ GMFM- 66 points in GMFCS I and $\geq 6.7$ points within each GMFCS level II-V, with $80 \%$ power, alpha $=0.05$. For secondary outcomes, we have differing power depending on sample characteristics, for example, two groups, evenly distributed, we will detect differences of $\geq 0.36 \mathrm{SD}$ with $\alpha=0.05,80 \%$ power.

\section{Statistical considerations}

Summary statistics will be described using either mean (SD) or median (25th-75th percentile) for continuous variables, according to distribution, or as frequency (percentage) for categorical variables. Cross-sectional associations will be assessed using linear regression for interval outcome data, with effect estimates presented as mean difference and 95\% CI, using logistic regression for binary outcome data, with effect estimates presented as ORs and 95\% CI, and using Poisson regression for count outcome data, with effect estimates presented as incidence rate ratios and 95\% CI. Longitudinal associations will be investigated using analyses that account 
for the multiple observations per participant. The particular analysis will be determined by data structure, for example, hierarchical mixed-effects models or generalised estimating equations. If hierarchical mixed-effects models are used then 'participant' will be included in the model as a random effect, to account for the possible non-independence of observations from the same participant. When building multivariable models, first univariable models including all potential variables of interest will be constructed. Variables will be selected for potential inclusion in multivariable models based on univariable significance at the $\mathrm{p}<0.2$ level. Multivariable models will be built in a stepwise manner with redundant variables eliminated using Akaike's and Schwarz's Bayesian criteria. In principle, multivariable models will be first constructed for the whole sample, then after stratification by GMFCS level. Interactions are investigated as appropriate.

Missing data were treated on a case-by-case basis depending on the observed pattern of missingness. For example, if data were 'missing at random', we will use multiple imputation methods, and if data are 'not missing at random', we will use pattern-mixture models. There was no global rule to account for multiple comparisons, instead adjustment for multiple comparisons will be made for each separate suite of analyses as appropriate, bearing in mind the type I and II error rates for each suite. Cost data will be standardised to current values. Cost data are typically skewed and therefore will be tested for normality and transformed using a log, gamma or another appropriate link function for the multivariable analysis.

Complex multivariable analyses accounting for attrition, if required, will be conducted by a biostatistician. The precise analyses will be determined by data structure. In principle, hierarchical mixed-effects models will be performed for the whole population then by GMFCS level. First, univariable then multivariable analyses will be undertaken. Variables included as fixed effects in the final multivariate model will be selected based on univariate results if $p<0.2$. Redundant variables are eliminated from multivariable models using Akaike's and Schwarz's Bayesian criteria. All models include a random intercept and slope (time effect) for each participant, accounting for the non-independence in repeated measures from the same participant and allowing for heterogeneity between participants. For Hypothesis $1{ }^{1}$, we will first undertake univariable analyses of the association between quantitative MRI (primary), motor capacity (primary), performance and potential confounding variables (eg, gender) at 2-5 years with motor capacity at $8-12$ years using a mixed-effects model with data grouped by individual participants to account for (up to 3) repeated measures between 2 and 5 years. Then all variables significant at $\mathrm{p}<0.2$ will be included in the prediction model, before being investigated for elimination. Interactions will be investigated as appropriate. Missing data will be treated on a case-by-case basis using MAR (multiple imputation algorithms) or NMAR (using pattern-mixture models). Adjustment for multiple comparisons will be made for each separate analyses mindful of type I and II error rates, as is standard practice.

\section{Author affiliations}

${ }^{1}$ Queensland Cerebral Palsy and Rehabilitation Research Centre (QCPRRC), The University of Queensland, Brisbane, Queensland, Australia

${ }^{2}$ Queensland Paediatric Rehabilitation Service, Lady Cilento Children's Hospital, Brisbane, Queensland, Australia

${ }^{3}$ Children's Nutrition Research Centre, The University of Queensland, Brisbane, Queensland, Australia

${ }^{4}$ School of Health and Rehabilitation Sciences, The University of Queensland, Brisbane, Queensland, Australia

${ }^{5}$ Institute of Health and Biomedical Innovation, Queensland University of Technology, Brisbane, Queensland, Australia

${ }^{6}$ Menzies Health Institute Queensland, Griffith University, Gold Coast, Queensland, Australia

${ }^{7}$ CSIRO Australian e-Health Research Centre, Canberra, Australia

${ }^{8}$ Medical Imaging, Diagnostic and Interventional Neuroradiology, Royal Brisbane and Women's Hospital, Brisbane, Queensland, Australia

${ }^{9}$ Queensland Children's Motion Analysis Service, Lady Cilento Children's Hospital,

Brisbane, Queensland, Australia

${ }^{10}$ Clinical Governance, Education and Research, Gold Coast Health, Brisbane, Queensland, Australia

${ }^{11}$ Centre for Clinical Research, The University of Queensland, Brisbane, Queensland, Australia

${ }^{12}$ Department of Developmental Neuroscience, Instituto Di Ricovero E Cura A

Carattere Scientifico (IRCCS), Pisa, Italy

${ }^{13}$ Department of Paediatric Orthopaedics, The Mater Health Services, Brisbane, Queensland, Australia

${ }^{14}$ Norwich Medical School, University of East Anglia, Norwich, UK

${ }^{15}$ School of Pharmacy, The University of Queensland, Brisbane, Queensland, Australia

Contributors $\mathrm{RB}$ is the chief investigator and together with chief investigators PSWD, JZ, ST, LB, RW, SR, KW, JAW, KB and Associate Investigators KB, CC, JW, PE, MK, LC, LS, KW, AC, AG conceptualised, designed and established this research study and successfully obtained study funding for a National Health and Medical Research Council (NHMRC) partnership grant. RP, KB, CD, S0, SR, DB, AB, SF and AG were responsible for the selection of particular assessments. JAW conceptualised the inclusion of quality of life instruments for children and carers able to support economic evaluation. RB, SR, SF and AG were responsible for conceptualising the brain MRI protocol and analysis. RB was responsible for ethics applications and reporting. RB, KB, LB, CC, PE, LS, LC, KW, DB, KB, CD, SO, SER, CF, AB, KM and OL were responsible for recruitment and data collection. RB drafted the manuscript with input from all the coauthors. All authors have agreed the final version of the manuscript and were involved in the decision to submit the manuscript. There is no financial support for the authors regarding this manuscript. The external funding agencies (NHMRC) have provided funds for the conduct of the study but will not be involved manuscript preparation, decisions to publish or the interpretation of results arising from the study.

Competing interests None declared.

Patient consent Yes.

Ethics approval Children's Health Queensland Hospital and Health Service Human Research Ethics Committee (HREC/14/QRCH/329) and the University of Queensland's Human Research Ethics Committees (2014001487).

Provenance and peer review Not commissioned; externally peer reviewed.

Open Access This is an Open Access article distributed in accordance with the terms of the Creative Commons Attribution (CC BY 4.0) license, which permits others to distribute, remix, adapt and build upon this work, for commercial use, provided the original work is properly cited. See: http://creativecommons.org/ licenses/by/4.0/

(C) Article author(s) (or their employer(s) unless otherwise stated in the text of the article) 2017. All rights reserved. No commercial use is permitted unless otherwise expressly granted. 


\section{REFERENCES}

1. Rosenbaum P, Paneth $N$, Leviton A, et al. A report: the definition and classification of cerebral palsy April 2006. Dev Med Child Neurol Suppl 2007;109:480.

2. Graham HK. Absence of reference to progressive musculoskeletal pathology in definition of cerebral palsy. Dev Med Child Neurol 2006;48:78-9.

3. Hanna SE, Rosenbaum PL, Bartlett DJ, et al. Stability and decline in gross motor function among children and youth with cerebral palsy aged 2 to 21 years. Dev Med Child Neurol 2009;51:295-302.

4. Australian Cerebral Palsy Register Group. 2013. Report of the Australian Cerebral Palsy Register. 2006.

5. Jordan R, David M, Pareezer L, et al. Relationship between health resource cost and performance outcomes in preschool age children with cerebral palsy: an economic analysis. Dev Med Child Neurol 2013;55(s3):6.

6. Novak I, Hines M, Goldsmith S, et al. Clinical prognostic messages from a systematic review on cerebral palsy. Pediatrics 2012;130:e12 85-e1312.

7. Bodimeade HL, Whittingham K, Lloyd O, et al. Executive function in children and adolescents with unilateral cerebral palsy. Dev Med Child Neurol 2013;55:926-33.

8. Fiori S, Cioni G, Klingels $\mathrm{K}$, et al. Reliability of a novel, semiquantitative scale for classification of structural brain magnetic resonance imaging in children with cerebral palsy. Dev Med Child Neurol 2014;56:839-45.

9. Australian Bureau of Statistics. Sport and Recreation: A Statistical Overview, Australia. Canberra, 2006.

10. Access EconomicsThe Economic impact of Cerebral Palsy in Australia in 2007.2008.

11. Krägeloh-Mann I, Horber V. The role of magnetic resonance imaging in elucidating the pathogenesis of cerebral palsy: a systematic review. Dev Med Child Neurol 2007;49:144-51.

12. Arnfield E, Guzzetta A, Boyd R. Relationship between brain structure on magnetic resonance imaging and motor outcomes in children with cerebral palsy: a systematic review. Res Dev Disabil 2013;34:2234-50.

13. Krägeloh-Mann I. Imaging of early brain injury and cortical plasticity. Exp Neurol 2004;190 Suppl 1(Suppl 1):84-90.

14. Boyd RN, Jordan R, Pareezer L, et al. Australian Cerebral Palsy Child Study: protocol of a prospective population based study of motor and brain development of preschool aged children with cerebral palsy. BMC Neurol 2013;13:57.

15. Pym A, Ware R, Jordan R, et al. Early predictors of hip displacement by school age in young children with cerebral palsy. Dev Med Child Neurol 2012;54(s6):61.

16. Whittingham K, Fahey M, Rawicki B, et al. The relationship between motor abilities and early social development in a preschool cohort of children with cerebral palsy. Res Dev Disabil 2010;31:1346-51.

17. Coleman A, Weir KA, Ware RS, et al. Relationship between communication skills and gross motor function in preschoolaged children with cerebral palsy. Arch Phys Med Rehabil 2013;94:2210-7.

18. Stephenson J, Bauman A, Armstrong T, et al. 2000. The Costs of Illness Attributable to Physical Inactivity in Australia. Canberra, Australia: Commonwealth Department of Health and Aged Care and the Australian Sports Commission.

19. Hagglund G, Andersson S, Duppe $\mathrm{H}$, et al. Prevention of dislocation of the hip in children with cerebral palsy: the first ten years of a population-based prevention programme. J Bone Joint Surg 2005;87:95-101.

20. Hägglund $G$, Wagner P. Spasticity of the gastrosoleus muscle is related to the development of reduced passive dorsiflexion of the ankle in children with cerebral palsy. Acta Orthop 2011;82:744-8.

21. Uddenfeldt Wort U, Nordmark E, Wagner P, et al. Fractures in children with cerebral palsy: a total population study. Dev Med Child Neurol 2013;55:821-6.

22. Westbom L, Lundkvist Josenby A, Wagner P, et al. Growth in Children with Cerebral Palsy during five years after Selective Dorsal Rhizotomy: a practice-based study. BMC Neurol 2010;10:57.

23. Wynter M, Gibson N, Kentish M, et al. The Consensus Statement on Hip Surveillance for Children with Cerebral Palsy: Australian Standards of Care. J Pediatr Rehabil Med 2011;4:183-95.

24. Kentish M, Wynter M, Snape N, et al. Five-year outcome of statewide hip surveillance of children and adolescents with cerebral palsy. J Pediatr Rehabil Med 2011;4:205-17.

25. Weil E, Wachterman M, McCarthy EP, et al. Obesity among adults with disabling conditions. JAMA 2002;288:1265-8.

26. Bell KL, Boyd RN, Tweedy SM, et al. A prospective, longitudinal study of growth, nutrition and sedentary behaviour in young children with cerebral palsy. BMC Public Health 2010;10:179.
27. Walker JL, Bell KL, Boyd RN, et al. Energy requirements in preschool-age children with cerebral palsy. Am J Clin Nutr 2012;96:1309-15.

28. Walker JL, Bell KL, Boyd RN, et al. Validation of a modified threeday weighed food record for measuring energy intake in preschoolaged children with cerebral palsy. Clin Nutr 2013;32:426-31.

29. Walker JL, Bell KL, Stevenson RD, et al. Relationships between dietary intake and body composition according to gross motor functional ability in preschool-aged children with cerebral palsy. Ann Nutr Metab 2012:61:349-57.

30. Oftedal S, Bell KL, Mitchell LE, et al. A systematic review of the clinimetric properties of habitual physical activity measures in young children with a motor disability. Int J Pediatr 2012;2012:1-12.

31. Benfer KA, Weir KA, Bell KL, et al. Oropharyngeal dysphagia and gross motor skills in children with cerebral palsy. Pediatrics 2013;131:e1553-e1562.

32. Weir KA, Bell KL, Caristo F, et al. Reported eating ability of young children with cerebral palsy: is there an association with gross motor function? Arch Phys Med Rehabil 2013;94:495-502.

33. Benfer KA, Weir KA, Bell KL, et al. Oropharyngeal dysphagia in preschool children with cerebral palsy: oral phase impairments. Res Dev Disabil 2014;35:3469-81.

34. Oftedal S, Bell KL, Davies PS, et al. Relationship between daily physical activity performance and functional mobility capacity and performance in toddlers with cerebral palsy. Dev Med Child Neurol 2013:s55.

35. Keawutan P, Bell K, Davies PS, et al. Systematic review of the relationship between habitual physical activity and motor capacity in children with cerebral palsy. Res Dev Disabil 2014;35:1301-9.

36. Barrett RS, Lichtwark GA. Gross muscle morphology and structure in spastic cerebral palsy: a systematic review. Dev Med Child Neurol 2010;52:794-804.

37. Graham HK. Botulinum toxin type A management of spasticity in the context of orthopaedic surgery for children with spastic cerebral palsy. Eur J Neurol 2001;8 Suppl 5(Suppl 5):30-9.

38. Bache CE, Selber P, Graham HK. (ii) The management of spastic diplegia. Curr Orthop 2003;17:88-104.

39. Opheim A, Jahnsen R, Olsson E, et al. Balance in relation to walking deterioration in adults with spastic bilateral cerebral palsy. Phys Ther 2012;92:279-88.

40. Barber L, Barrett R, Lichtwark G. Passive muscle mechanical properties of the medial gastrocnemius in young adults with spastic cerebral palsy. J Biomech 2011;44:2496-500.

41. Barber L, Hastings-Ison T, Baker R, et al. Medial gastrocnemius muscle volume and fascicle length in children aged 2 to 5 years with cerebral palsy. Dev Med Child Neurol 2011;53:543-8.

42. Barber L, Barrett R, Lichtwark G. Medial gastrocnemius muscle fascicle active torque-length and Achilles tendon properties in young adults with spastic cerebral palsy. J Biomech 2012;45:2526-30.

43. Wren TA, Cheatwood AP, Rethlefsen SA, et al. Achilles tendon length and medial gastrocnemius architecture in children with cerebral palsy and equinus gait. J Pediatr Orthop 2010;30:479-84.

44. Perry J, Hoffer MM, Giovan P, et al. Gait analysis of the triceps surae in cerebral palsy. A preoperative and postoperative clinical and electromyographic study. J Bone Joint Surg Am 1974;56:511-20.

45. Eek MN, Beckung E. Walking ability is related to muscle strength in children with cerebral palsy. Gait Posture 2008;28:366-71.

46. Barber LA, Read F, Lovatt Stern J, et al. Medial gastrocnemius muscle volume in ambulant children with unilateral and bilateral cerebral palsy aged 2 to 9 years. Dev Med Child Neurol 2016;58:1146-52.

47. In: Gage JR, Schwartz MH, Koop SE, Novacheck TF, et al. The identification and treatment of Gait problems in Cerebral Palsy. 2nd ed. London: MacKeith Press, 2009.

48. Winters TF, Gage JR, Hicks R. Gait patterns in spastic hemiplegia in children and young adults. J Bone Joint Surg Am 1987;69:437-41.

49. Howard J, Soo B, Graham HK, et al. Cerebral palsy in Victoria: motor types, topography and gross motor function. J Paediatr Child Health 2005;41(9-10):479-83.

50. Palisano R, Rosenbaum P, Walter S, et al. Development and reliability of a system to classify gross motor function in children with cerebral palsy. Dev Med Child Neurol 1997;39:214-23.

51. Wood E, Rosenbaum P. The gross motor function classification system for cerebral palsy: a study of reliability and stability over time. Dev Med Child Neurol 2000;42:292-6.

52. Oeffinger DJ, Tylkowski CM, Rayens MK, et al. Gross Motor Function Classification System and outcome tools for assessing ambulatory cerebral palsy: a multicenter study. Dev Med Child Neurol 2004;46:311-9. 
53. Palisano RJ, Rosenbaum P, Bartlett D, et al. Content validity of the expanded and revised Gross Motor Function Classification System. Dev Med Child Neurol 2008;50:744-50.

54. Gorter JW, Rosenbaum PL, Hanna SE, et al. Limb distribution, motor impairment, and functional classification of cerebral palsy. Dev Med Child Neurol 2004;46:461-7.

55. Surveillance of Cerebral Palsy in Europe. Surveillance of cerebral palsy in Europe: a collaboration of cerebral palsy surveys and registers. Surveillance of Cerebral Palsy in Europe (SCPE). Dev Med Child Neurol 2000;42:816-24.

56. Monbaliu E, Ortibus E, De Cat J, et al. The Dyskinesia Impairment Scale: a new instrument to measure dystonia and choreoathetosis in dyskinetic cerebral palsy. Dev Med Child Neuro/ 2012;54:278-83.

57. Monbaliu E, de Cock P, Ortibus E, et al. Clinical patterns of dystonia and choreoathetosis in participants with dyskinetic cerebral palsy. Dev Med Child Neurol 2016;58:138-44.

58. Harvey A, Graham HK, Morris ME, et al. The Functional Mobility Scale: ability to detect change following single event multilevel surgery. Dev Med Child Neurol 2007;49:603-7.

59. Harvey AR, Morris ME, Graham HK, et al. Reliability of the functional mobility scale for children with cerebral palsy. Phys Occup Ther Pediatr 2010;30:139-49.

60. Rodda J, Graham HK. Classification of gait patterns in spastic hemiplegia and spastic diplegia: a basis for a management algorithm. Eur J Neurol 2001;8 Suppl 5:98-108.

61. Dobson F, Morris ME, Baker R, et al. Gait classification in children with cerebral palsy: a systematic review. Gait Posture 2007;25:140-52.

62. Dobson F, Morris ME, Baker R, et al. Clinician agreement on gait pattern ratings in children with spastic hemiplegia. Dev Med Child Neurol 2006;48:429-35.

63. Stott NS, Atherton WG, Mackey AH, et al. The reliability and validity of assessment of sagittal plane deviations in children who have spastic diplegia. Arch Phys Med Rehabil 2005;86:2337-41.

64. Eliasson AC, Krumlinde-Sundholm L, Rösblad B, et al. The Manual Ability Classification System (MACS) for children with cerebral palsy: scale development and evidence of validity and reliability. Dev Med Child Neurol 2006;48:549-54.

65. Morris C, Kurinczuk JJ, Fitzpatrick R, et al. Reliability of the manual ability classification system for children with cerebral palsy. Dev Med Child Neurol 2006;48:950-3.

66. Hidecker MJ, Paneth N, Rosenbaum PL, et al. Developing and validating the Communication Function Classification System for individuals with cerebral palsy. Dev Med Child Neurol 2011;53:704-10.

67. Barty E, Caynes K, Johnston LM. Development and reliability of the Functional Communication Classification System for children with cerebral palsy. Dev Med Child Neurol 2016;58:1036-41.

68. Pennington L, Virella D, Mjøen T, et al. Development of The Viking Speech Scale to classify the speech of children with cerebral palsy. Res Dev Disabil 2013;34:3202-10.

69. Sellers D, Mandy A, Pennington L, et al. Development and reliability of a system to classify the eating and drinking ability of people with cerebral palsy. Dev Med Child Neurol 2014;56:245-51.

70. Ashwal S, Russman BS, Blasco PA, et al. Practice Parameter: diagnostic assessment of the child with Cerebral Palsy. Neurology 2004:62:851-63.

71. Holmes CJ, Hoge R, Collins L, et al. Enhancement of MR images using registration for signal averaging. $J$ Comput Assist Tomogr 1998:22:324-33.

72. Fiori S, Cioni G, Klingels K, et al. Reliability of a novel, semiquantitative scale for classification of structural brain magnetic resonance imaging in children with cerebral palsy. Dev Med Child Neurol 2014;56:839-45.

73. Fiori S, Guzzetta A, Pannek K, et al. Validity of semiquantitative scale for brain MRI in unilateral cerebral palsy due to periventricular white matter lesions: Relationship with hand sensorimotor function and structural connectivity. Neuroimage Clin 2015;8:104-9.

74. Pannek K, Raffelt D, Bell C, et al. HOMOR: higher order model outlier rejection for high b-value MR diffusion data. Neuroimage 2012:63:835-42.

75. Tournier JD, Calamante F, Connelly A. Robust determination of the fibre orientation distribution in diffusion MRI: non-negativity constrained super-resolved spherical deconvolution. Neuroimage 2007;35:1459-72

76. Pannek K, Mathias JL, Bigler ED, et al. An automated strategy for the delineation and parcellation of commissural pathways suitable for clinical populations utilising high angular resolution diffusion imaging tractography. Neuroimage 2010;50:1044-53.
77. Rose S, Rowland T, Pannek K, et al. Structural hemispheric asymmetries in the human precentral gyrus hand representation. Neuroscience 2012;210:211-21.

78. Cornell MS, Hatrick NC, Boyd R, et al. The hip in children with cerebral palsy. Predicting the outcome of soft tissue surgery. Clin Orthop Relat Res 1997;340:165-71.

79. Faraj S, Atherton WG, Stott NS. Inter- and intra-measurer error in the measurement of Reimers' hip migration percentage. J Bone Joint Surg Br 2004;86:434-7.

80. Parrott J, Boyd RN, Dobson F, et al. Hip displacement in spastic cerebral palsy: repeatability of radiologic measurement. J Pediatr Orthop 2002;22:660-7.

81. Scrutton D, Baird G. Surveillance measures of the hips of children with bilateral cerebral palsy. Arch Dis Child 1997;76:381-4.

82. Scrutton D, Baird G, Smeeton N. Hip dysplasia in bilateral cerebral palsy: incidence and natural history in children aged 18 months to 5 years. Dev Med Child Neurol 2001;43:586-600.

83. Boyd RN, Dobson F, Parrott J, et al. The effect of botulinum toxin type $A$ and a variable hip abduction orthosis on gross motor function: a randomized controlled trial. Eur J Neurol 2001;8 Suppl 5:109-19.

84. Stevenson RD. Use of segmental measures to estimate stature in children with cerebral palsy. Arch Pediatr Adolesc Med 1995;149:658-62.

85. Ogden CL, Kuczmarski RJ, Flegal KM, et al. Centers for Disease Control and Prevention 2000 growth charts for the United States: improvements to the 1977 National Center for Health Statistics version. Pediatrics 2002;109:45-60.

86. Russell D, Rosenbaum P, Avery L, et al. Gross motor function measure (GMFM-66 and GMFM-88) user's manual, 2002 MacKeith Press London.

87. Holmefur M, Krumlinde-Sundholm L, Bergström J, et al. Longitudinal development of hand function in children with unilateral cerebral palsy. Dev Med Child Neurol 2010;52:352-7.

88. Elvrum A-KG. Assessment of hand function in children with bilateral cerebral palsy: development and measurement properties of outcome measures and classifications. Trondheim, Norway: Norwegian University of Science and Technology, 2016.

89. Oldfield RC. The assessment and analysis of handedness: the Edinburgh inventory. Neuropsychologia 1971;9:97-113.

90. Auld ML, Boyd RN, Moseley GL, et al. Impact of tactile dysfunction on upper-limb motor performance in children with unilateral cerebral palsy. Arch Phys Med Rehabil 2012;93:696-702.

91. Sakzewski L, Ziviani J, Boyd R. The relationship between unimanual capacity and bimanual performance in children with congenital hemiplegia. Dev Med Child Neurol 2010;52:811-6.

92. Hägglund G, Lauge-Pedersen H, Wagner P. Characteristics of children with hip displacement in cerebral palsy. BMC Musculoskelet Disord 2007;8:101.

93. Wynter M, Gibson N, Kentish M, et al. The development of Australian Standards of Care for Hip Surveillance in Children with Cerebral Palsy: how did we reach consensus? J Pediatr Rehabil Med 2011;4:171-82.

94. Reimers J, Bialik V. Influence of femoral rotation on the radiological coverage of the femoral head in children. Pediatr Radiol $1981 ; 10: 215-8$

95. Foroohar A, McCarthy JJ, Yucha D, et al. Head-shaft angle measurement in children with cerebral palsy. $J$ Pediatr Orthop 2009;29:248-50.

96. Hilgenreiner $\mathrm{H}$. Early diagnosis and early treatment of congenital dislocation of the hip. Med Klin 1925;21:1385-425.

97. Robin J, Graham HK, Selber P, et al. Proximal femoral geometry in cerebral palsy: a population-based cross-sectional study. J Bone Joint Surg Br 2008;90:1372-9.

98. Birkenmaier C, Jorysz G, Jansson V, et al. Normal development of the hip: a geometrical analysis based on planimetric radiography. $J$ Pediatr Orthop B 2010;19:1-8.

99. Haike H, Breuckmann G, Schulze H. [Surgical treatment of so-called congenital hip dislocation]. Arch Orthop Unfallchir 1969;66:277-85.

100. Morrissy RT, Goldsmith GS, Hall EC, et al. Measurement of the Cobb angle on radiographs of patients who have scoliosis. Evaluation of intrinsic error. J Bone Joint Surg Am 1990;72:320-7.

101. Henderson RC, Henderson BA, Kecskemethy HH, et al. Adaptation of the lateral distal femur DXA scan technique to adults with disabilities. J Clin Densitom 2015;18:102-8.

102. Henderson RC, Lark RK, Newman JE, et al. Pediatric reference data for dual X-ray absorptiometric measures of normal bone density in the distal femur. AJR Am J Roentgenol 2002;178:439-43.

103. Mueske NM, Chan LS, Wren TA. Reliability of lateral distal femur dual-energy X-ray absorptiometry measures. J Clin Densitom 2014;17:522-7. 
104. Zemel BS, Stallings VA, Leonard MB, et al. Revised pediatric reference data for the lateral distal femur measured by Hologic Discovery/Delphi dual-energy X-ray absorptiometry. J Clin Densitom 2009;12:207-18.

105. Carter DR, Bouxsein ML, Marcus R. New approaches for interpreting projected bone densitometry data. J Bone Miner Res 1992;7:137-45.

106. Australian Radiation Protection and Nuclear Safety Agency. 2005. Code of Practice: Exposure of Humans to lonizing Radiation for Research Purposes. Radiation Protection Series Publication No. 8.

107. Duke PM, Litt IF, Gross RT. Adolescents' self-assessment of sexual maturation. Pediatrics 1980;66:918-20.

108. Varni JW, Thompson KL, Hanson V. The Varni/Thompson Pediatric Pain Questionnaire. I. Chronic musculoskeletal pain in juvenile rheumatoid arthritis. Pain 1987;28:27-38.

109. Gragg RA, Rapoff MA, Danovsky MB, et al. Assessing chronic musculoskeletal pain associated with rheumatic disease: further validation of the pediatric pain questionnaire. J Pediatr Psychol 1996;21:237-50.

110. Kainz H, Modenese L, Lloyd DG, et al. Joint kinematic calculation based on clinical direct kinematic versus inverse kinematic gait models. J Biomech 2016;49:1658-69.

111. Steele KM, Rozumalski A, Schwartz MH. Muscle synergies and complexity of neuromuscular control during gait in cerebral palsy. Dev Med Child Neurol 2015;57:1176-82.

112. Delp SL, Anderson FC, Arnold AS, et al. OpenSim: open-source software to create and analyze dynamic simulations of movement. IEEE Trans Biomed Eng 2007;54:1940-50.

113. Baker R, McGinley JL, Schwartz MH, et al. The gait profile score and movement analysis profile. Gait Posture 2009;30:265-9.

114. Lichtwark GA, Wilson AM. Interactions between the human gastrocnemius muscle and the Achilles tendon during incline, level and decline locomotion. J Exp Biol 2006;209:4379-88.

115. Cronin NJ, Carty CP, Barrett RS, et al. Automatic tracking of medial gastrocnemius fascicle length during human locomotion. J Appl Physiol 2011;111:1491-6.

116. Gillett JG, Barrett RS, Lichtwark GA. Reliability and accuracy of an automated tracking algorithm to measure controlled passive and active muscle fascicle length changes from ultrasound. Comput Methods Biomech Biomed Engin 2013;16:678-87.

117. Barber $L$, Barrett R, Lichtwark $G$. Validation of a freehand $3 D$ ultrasound system for morphological measures of the medial gastrocnemius muscle. J Biomech 2009;42:1313-9.

118. Verschuren $\mathrm{O}$, Ketelaar $\mathrm{M}$, Keefer $\mathrm{D}$, et al. Identification of a core set of exercise tests for children and adolescents with cerebral palsy: a Delphi survey of researchers and clinicians. Dev Med Child Neurol 2011;53:449-56.

119. Maher CA, Williams MT, Olds TS. The six-minute walk test for children with cerebral palsy. Int J Rehabil Res 2008;31:185-8.

120. Li AM, Yin J, Au JT, et al. Standard reference for the six-minute-walk test in healthy children aged 7 to 16 years. Am J Respir Crit Care Med 2007;176:174-80.

121. ATS Committee on Proficiency Standards for Clinical Pulmonary Function Laboratories. ATS statement: guidelines for the six-minute walk test. Am J Respir Crit Care Med 2002;166:111-7.

122. Verschuren $\mathrm{O}$, Takken $\mathrm{T}$, Ketelaar $\mathrm{M}$, et al. Reliability for running tests for measuring agility and anaerobic muscle power in children and adolescents with cerebral palsy. Pediatr Phys Ther 2007;19:108-15.

123. Verschuren $\mathrm{O}$, Bongers $\mathrm{BC}$, Obeid $\mathrm{J}$, et al. Validity of the muscle power sprint test in ambulatory youth with cerebral palsy. Pediatr Phys Ther 2013:25:25-8.

124. Thompson P, Beath T, Bell J, et al. Test-retest reliability of the 10-metre fast walk test and 6-minute walk test in ambulatory school-aged children with cerebral palsy. Dev Med Child Neurol 2008;50:370-6.

125. Verschuren O, Ketelaar M, Takken T, et al. Reliability of hand-held dynamometry and functional strength tests for the lower extremity in children with Cerebral Palsy. Disabil Rehabil 2008;30:1358-66.

126. Trost SG, Mclver KL, Pate RR. Conducting accelerometer-based activity assessments in field-based research. Med Sci Sports Exerc 2005;37(11 Suppl):S531-S543.

127. Clanchy KM, Tweedy SM, Boyd R. Measurement of habitual physical activity performance in adolescents with cerebral palsy: a systematic review. Dev Med Child Neurol 2011:53:499-505.

128. Clanchy KM, Tweedy SM, Boyd RN, et al. Validity of accelerometry in ambulatory children and adolescents with cerebral palsy. Eur $J$ Appl Physiol 2011:111:2951-9.

129. National Health and Medical Research Council. 2005. Nutrient Reference Values for Australia and New Zealand. Canberra, Australia: Department of Health and Ageing.
130. Taylor C, Lamparello B, Kruczek K, et al. Validation of a food frequency questionnaire for determining calcium and vitamin $D$ intake by adolescent girls with anorexia nervosa. J Am Diet Assoc 2009;109:479-85

131. Harrison SL, Buettner PG, Maclennan R. The North Queensland "Sun-Safe Clothing" study: design and baseline results of a randomized trial to determine the effectiveness of sun-protective clothing in preventing melanocytic nevi. Am J Epidemiol 2005; $161: 536-45$

132. Sheppard J. Dysphagia Disorders survey and Dysphagia Management Staging Scale (Adult and Pediatric applications): User's Manual: Australian Edition. Ryde, NSW.: The Centre for Developmental Disability, 2003.

133. Sheppard JJ, Hochman R, Baer C. The dysphagia disorder survey: validation of an assessment for swallowing and feeding function in developmental disability. Res Dev Disabil 2014;35:929-42.

134. Benfer KA, Weir KA, Bell KL, et al. Longitudinal cohort protocol study of oropharyngeal dysphagia: relationships to gross motor attainment, growth and nutritional status in preschool children with cerebral palsy. BMJ Open 2012;2:e001460.

135. Calis EA, Veugelers R, Sheppard JJ, et al. Dysphagia in children with severe generalized cerebral palsy and intellectual disability. Dev Med Child Neurol 2008;50:625-30.

136. Sheppard JJ, Liou J, Hochman R, et al. Nutritional correlates of dysphagia in individuals institutionalized with mental retardation. Dysphagia 1988;3:85-9.

137. Skuse D, Stevenson J, Reilly S, et al. Schedule for oralmotor assessment (SOMA): methods of validation. Dysphagia 1995; 10:192-202

138. Weir K, McMahon S, Barry L, et al. Clinical signs and symptoms of oropharyngeal aspiration and dysphagia in children. Eur Respir $J$ 2009;33:604-11.

139. DeMatteo C, Matovich D, Hjartarson A. Comparison of clinical and videofluoroscopic evaluation of children with feeding and swallowing difficulties. Dev Med Child Neurol 2005;47:149-57.

140. Lefton-Greif MA, McGrath-Morrow SA. Deglutition and respiration: development, coordination, and practical implications. Semin Speech Lang 2007;28:166-79.

141. Thomas-Stonell N, Greenberg J. Three treatment approaches and clinical factors in the reduction of drooling. Dysphagia 1988;3:73-8.

142. Benfer KA, Weir KA, Bell KL, et al. Longitudinal Study of Oropharyngeal Dysphagia in Preschool Children With Cerebral Palsy. Arch Phys Med Rehabil 2016;97:552-60.

143. Lefton-Greif MA, Okelo SO, Wright JM, et al. Impact of children's feeding/swallowing problems: validation of a new caregiver instrument. Dysphagia 2014;29:671-7.

144. Semel E, Wiig E, Secord W. Clinical Evaluation of Language Fundamentals. 4th edition. Marrickville: Pearsons, 2006.

145. Fenson L, Marchmann V, Thal D, et al. McArthur Bates Communicative Development Inventory. 2nd edition. Baltimore: Paul H Brookes Publishing Co, 2007.

146. Hayden D, Square P. Verbal Motor Performance Assessment for Children: examiner's Manual. USA.: Psychological Corporation, 1999.

147. Haley SM, Coster WJ, Dumas HM, et al. Pediatric Evaluation of Disability Inventory Computer Adaptive Test, Development, Standardization and Administration Manual Version 1.3.6 Boston University. Boston 2012. MA: CRECare LLC.

148. Haley SM, Andrellos PJ, Coster W, et al; Pediatric valuation of isability inventory: , 1992.

149. Coster W, Bedell G, Law M, et al. Psychometric evaluation of the Participation and Environment Measure for Children and Youth. Dev Med Child Neurol 2011;53:1030-7.

150. Goodman R. The Strengths and Difficulties Questionnaire: a research note. J Child Psychol Psychiatry 1997;38:581-6.

151. Goodman R. The extended version of the Strengths and Difficulties Questionnaire as a guide to child psychiatric caseness and consequent burden. J Child Psychol Psychiatry 1999;40:791-9.

152. Goodman R. Psychometric properties of the strengths and difficulties questionnaire. J Am Acad Child Adolesc Psychiatry 2001;40:1337-45.

153. Australian Government. 2009. A Snapshot of Early Childhood Development in Australia 2012 - AEDI National Report. Canberra: The Australian Government.

154. Australian Curriculum Assessment and Reporting Authority. Measurement Framework for schooling in Australia. Sydney, Australia: Education Council, 2013.

155. Cotton SM, Kiely PM, Crewther DP, et al. A normative and reliability study for the Raven's Coloured Progressive Matrices for primary school aged children from Victoria, Australia. Pers Individ Dif 2005;39:647-59. 
156. Pueyo R, Junqué C, Vendrell P, et al. Raven's Coloured Progressive Matrices as a measure of cognitive functioning in Cerebral Palsy. $J$ Intellect Disabil Res 2008;52:437-45.

157. Gioia G, Isquith P, Guy S, et al. Behavior Rating Inventory of Executive function. Lutz, Florida: Parnic, 2000.

158. Reynolds C, Kamphaus R. Behavior Assessment System for children. Circle Pines. American Guidance Service 1992.

159. Achenbach T, . Integrative Guide to the 1991 CBCL/4-18, YSR, and TRF profiles. Burlington, VT: University of Vermont, 1991.

160. Conners CK. Conner's Rating Scales-Revised Technical Manual. Toronto: Multi-Health Systems, 1997.

161. Allison C, Auyeung B, Baron-Cohen S. Toward brief "Red Flags" for autism screening: The Short Autism Spectrum Quotient and the Short Quantitative Checklist for Autism in toddlers in 1,000 cases and 3,000 controls [corrected]. J Am Acad Child Adolesc Psychiatry 2012;51:202-12.

162. Newman CJ, O'Regan M, Hensey O. Sleep disorders in children with cerebral palsy. Dev Med Child Neurol 2006;48:564-8.

163. Bruni O, Ottaviano S, Guidetti V, et al. The Sleep Disturbance Scale for Children (SDSC). Construction and validation of an instrument to evaluate sleep disturbances in childhood and adolescence. J Sleep Res 1996;5:251-61.

164. Davis E, Shelly A, Waters E, et al. Quality of life of adolescents with cerebral palsy: perspectives of adolescents and parents. Dev Med Child Neurol 2009;51:193-9.

165. Shelly A, Davis E, Waters E, et al. The relationship between quality of life and functioning for children with cerebral palsy. Dev Med Child Neurol 2008;50:199-203.

166. Waters E, Davis E, Mackinnon A, et al. Psychometric properties of the quality of life questionnaire for children with CP. Dev Med Child Neurol 2007;49:49-55.

167. Olree KS, Engsberg JR, Ross SA, et al. Changes in synergistic movement patterns after selective dorsal rhizotomy. Dev Med Child Neurol 2000;42:297-303.

168. Stevens K. Developing a descriptive system for a new preferencebased measure of health-related quality of life for children. Qual Life Res 2009;18:1105-13.
169. Stevens KJ. Working with children to develop dimensions for a preference-based, generic, pediatric, health-related quality-of-life measure. Qual Health Res 2010;20:340-51.

170. Stevens K. Assessing the performance of a new generic measure of health-related quality of life for children and refining it for use in health state valuation Applied Health Economics and Health Policy. 2011;9:157-69.

171. Brooks R. EuroQol: the current state of play. Health Policy 1996;37:53-72.

172. Herdman M, Gudex C, Lloyd A, et al. Development and preliminary testing of the new five-level version of EQ-5D (EQ-5D-5L). Qual Life Res 2011;20:1727-36.

173. Norman R, Cronin P, Viney R. A pilot discrete choice experiment to explore preferences for EQ-5D-5L health states. Appl Health Econ Health Policy 2013;11:287-98.

174. Australian Bureau of Statistics. Census of population and housing: socio-economic indexes for areas (SEIFA), Australia. 2014.

175. Al-Janabi H, Coast J, Flynn TN. What do people value when they provide unpaid care for an older person? A meta-ethnography with interview follow-up. Soc Sci Med 2008;67:111-21.

176. Al-Janabi H, Flynn TN, Coast J. Estimation of a preference-based carer experience scale. Med Decis Making 2011;31:458-68.

177. Brehaut JC, Kohen DE, Raina P, et al. The health of primary caregivers of children with cerebral palsy: how does it compare with that of other Canadian caregivers? Pediatrics 2004;114:e182-e191.

178. Epstein NB, Baldwin LM, Bishop DS. THE McMASTER FAMILY ASSESSMENT DEVICE*. J Marital Fam Ther 1983;9:171-80.

179. Miller IW, Epstein NB, Bishop DS, et al. THE McMASTER FAMILY ASSESSMENT DEVICE: RELIABILITY AND VALIDITY*. J Marital Fam Ther 1985;11:345-56.

180. Byles J, Byrne C, Boyle MH, et al. Ontario Child Health Study: reliability and validity of the general functioning subscale of the McMaster Family Assessment Device. Fam Process 1988;27:97-104.

181. Rosenbaum PL, Walter SD, Hanna SE, et al. Prognosis for gross motor function in cerebral palsy: creation of motor development curves. JAMA 2002;288:1357-63. 